\title{
MicroRNA expression profiles and networks in placentas complicated with selective intrauterine growth restriction
}

\author{
HONG WEN, LU CHEN, JING HE and JUN LIN \\ Department of Obstetrics, The Women's Hospital, School of Medicine, \\ Zhejiang University, Hangzhou, Zhejiang 310006, P.R. China
}

Received February 3, 2017; Accepted August 24, 2017

DOI: $10.3892 / \mathrm{mmr} .2017 .7462$

\begin{abstract}
The microRNA (miRNA) profiles of placentas complicated with selective intrauterine growth restriction (sIUGR) are unknown. In the present study, the sIUGR-associated placental miRNA expression was investigated using microarray and confirmatory reverse transcriptase-quantitative polymerase chain reaction studies. Placenta samples around the individual insertion region for each umbilical cord were collected from monochorionic twins complicated with $(n=17)$ or without sIUGR (control, $n=16)$. miRNA profile analysis was performed on two sIUGR cases and one control using an Affymetrix microRNA 4.0 Array system. A total of 14 miRNAs were identified to be specifically differentially expressed (7 upregulated and 7 downregulated) among larger twins of sIUGR cases compared with smaller twins of sIUGR cases. The target genes of the identified miRNAs participate in organ size, cell differentiation, cell proliferation and migration. In addition, according to the miRNA-pathway network analysis, key miRNAs and pathways (transforming growth factor- $\beta$, mitogen-activated protein kinase and Wnt) were identified to be associated with the pathogenesis of sIUGR. To the best of our knowledge, the results of the current study have provided the most complete miRNA profiles and the most detailed miRNA regulatory networks of placental tissues complicated with sIUGR.
\end{abstract}

\section{Introduction}

MicroRNAs (miRNAs), 21-25 nucloeotide long non-coding RNA molecules, are highly ubiquitous and conserved across many species (1). miRNA binds to the 3'-untranslated region

Correspondence to: Dr Jun Lin or Dr Jing He, Department of Obstetrics, The Women's Hospital, School of Medicine, Zhejiang University, 1 Xueshi Road, Uptown, Hangzhou, Zhejiang 310006, P.R. China

E-mail: linjun1088@163.com

E-mail: hej@zju.edu.cn

Key words: microRNA, placenta, selective intrauterine growth restriction, microarray of target mRNA and silence gene expression by either translational repression or direct mRNA degradation (2). Human genome codes for more than 1,000 miRNAs, and each of them can potentially post-transcriptionally regulate a vast number of genes. By negatively regulating their mRNA targets, miRNA have been implicated in regulating a number of key cellular functions including cell migration, invasion, growth, differentiation and apoptosis $(3,4)$. miRNA expression has been detected expressed in diverse tissues, including placenta (5). Altered expression of miRNAs has been showed in pregnancy-specific diseases, such as preeclampsia, ectopic pregnancy, fetal growth restriction and intrauterine growth retardation (6).

Selective intrauterine growth restriction (sIUGR) is used to define cases with an estimated fetal weight (EFW) of below the 10 th percentile in one fetus $(7,8)$. sIUGR occurs in 10 to $15 \%$ of monochorionic (MC) twins and is associated with an increased risk of intrauterine fetal demise (IUFD) and neurological adverse outcome for both twins (9). The presence of vascular anastomoses, the localization of umbilical cord and the unequal placental sharing are associated with the development of sIUGR in monozygotic twins, which have identical inherited backgrounds (10-12), while the molecular mechanisms underlying the pathogenesis of sIUGR are still unclear. Studies have showed that several angiogenic and antiangiogenic factors [vascular endothelial growth factor receptor-1 (VEGFR-1), endoglin and fms-Like Tyrosine Kinase-1 (Flt-1)] are involved in the pathogenesis of twin pregnancies complicated by sIUGR (13-15). Unbalanced placental expression of imprinted genes such as PHLDA2 (16) and insulin-like growth factor 2 (IGF2) (17) may also contribute to the development of sIUGR. However, little is known about the dysregulated miRNAs in the placentas complicated sIUGR.

The aim of this study was to identify miRNA profiles in the placentas from pregnancies complicated by sIUGR. The placentas around the individual insertion region for each umbilical cord were collected and subjected for miRNA profile analysis using Affymetrix microRNA 4.0 Array System. We characterized 14 specific significant differentially expressed miRNAs (DEMs) in larger twin placenta compared to corresponding smaller twin placenta. The target genes of significantly changed miRNAs were predicted, and miRNA-Pathway network was established, which provided 
Table I. Primer sequence for qRT-PCR.

A, RT primer sequences.

miRNA

Primer sequence

has-miR-1

has-miR-370-3p

has-miR-5189-5p

has-miR-373-3p

has-miR-338-5p

has-miR-590-5p
5'-CTCAACTGGTGTCGTGGAGTCGGCAATTCAGTTGAGATGGGC-3'

5'-CTCAACTGGTGTCGTGGAGTCGGCAATTCAGTTGAGATGGGC-3'

5'-CTCAACTGGTGTCGTGGAGTCGGCAATTCAGTTGAGCCTGTC-3'

5'-CTCAACTGGTGTCGTGGAGTCGGCAATTCAGTTGAGACACCC-3'

5'-CTCAACTGGTGTCGTGGAGTCGGCAATTCAGTTGAGCACTCA-3'

5'-CTCAACTGGTGTCGTGGAGTCGGCAATTCAGTTGAGCTGCAC-3'

$\mathrm{B}, \mathrm{PCR}$ primer sequences

miRNA

has-miR-1

has-miR-370-3p

has-miR-5189-5p

has-miR-373-3p

has-miR-338-5p

has-miR-590-5p

U6

Universal reverse
Primer sequence

5'-ACACTCCAGCTGGGACATACTTCTTTATAT-3'
5'-ACACTCCAGCTGGGGCCTGCTGGGGTGGAA-3'
5'-ACACTCCAGCTGGGTCTGGGCACAGGCGGATG-3'
5'-ACACTCCAGCTGGGGAAGTGCTTCGATTTTG-3'
5'-ACACTCCAGCTGGGAACAATATCCTGGTGC-3'
5'-ACACTCCAGCTGGGGAGCTTATTCATAAAA-3'
5'-CTCGCTTCGGCAGCACA-3' and 5'-AACGCTTCACGAATTTGCGT-3'
5'-TGGTGTCGTGGAGTCG-3'

Table II. Identified DEMs.

\begin{tabular}{lrcc}
\hline Category & Up-regulated & Down-regulated & Total \\
\hline L1 vs. S1 & 84 & 46 & 130 \\
L2 vs. S2 & 107 & 41 & 148 \\
N1 vs. n2 & 50 & 83 & 133 \\
\hline
\end{tabular}

Fold change $>2, \mathrm{P}<0.05$.

comprehensive information on the molecular mechanisms of sIUGR.

\section{Materials and methods}

Collection of placenta samples. The study was performed with the approval of the Institutional Review Board of Zhejiang University. All participating women were given written, informed consent prior to the collection of samples. Thirty-three women were enrolled in this study, including 17 cases complicated with sIUGR and 16 cases with normal MC. The intertwin EFW discordance, calculated as [(larger twin-smaller twin)/larger twin], was above $20 \%$ and less than $5 \%$ for sIUGR and normal MC, respectively. Pregnancies complicated with twin-to-twin transfusion syndrome (TTTs), severe congenital anomalies and maternal complication were excluded from this study. The placentas around the individual insertion region for each umbilical cord were collected within $30 \mathrm{~min}$ after delivery. The tissue was excised from inside the placental lobules, avoiding both the maternal surface and

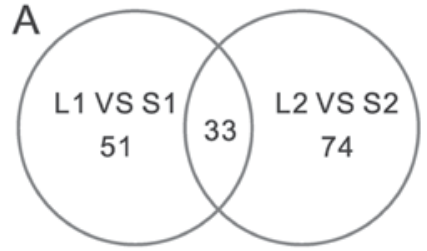

Up-regulated miRNAs

$\mathrm{B}$

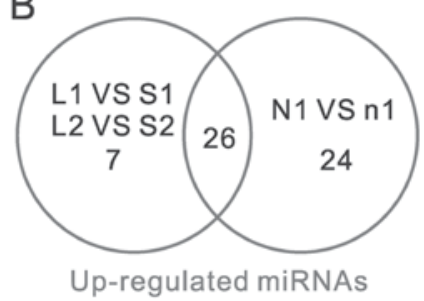

Down-regulated miRNAs

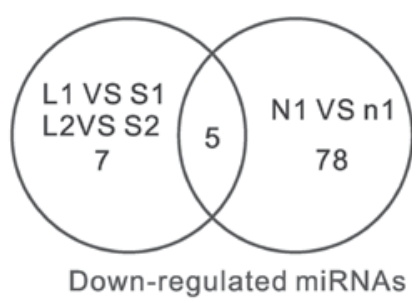

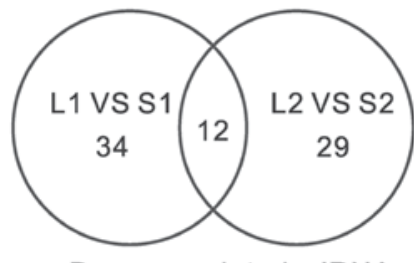

Figure 1. Venn-analysis of miRNAs. (A) Overlap of L1 vs. S1 and L2 vs. S2. (B) Overlap of (L1 vs. S1, L2 vs. S2) and N1 vs. n1. L1 and L2, placenta tissues supporting larger twins from case 1 and case 2 with sIUGR (intertwin EFW discordance was more than 25\%), respectively; S1 and S2, placenta tissues supporting smaller twins from case 1 and case 2 with sIUGR, respectively. N1 and n1, placenta tissues supporting larger and smaller twin form normal MC (intertwin EFW discordance was less than $5 \%$ ), respectively.

the amniotic membrane. The excised tissues were washed in sterilized ice-cold PBS to eliminate any blood and stored at $-80^{\circ} \mathrm{C}$ until they were used to isolate RNA. Placenta samples from two cases with sIUGR [larger twin (L1 and L2), smaller twin (S1 and S2)] and one cases with normal MC [larger twin (N1) and smaller twin (n1)] were used for miRNA profiling; Placenta samples from other 15 cases with sIUGR and other 
Table III. DEMs (L1 vs. S1).

\begin{tabular}{|c|c|c|c|c|c|}
\hline Regulation & $\begin{array}{c}\text { Systematic } \\
\text { name }\end{array}$ & $\begin{array}{c}\text { FC } \\
(\mathrm{L} 1 \text { vs. S1) }\end{array}$ & $\begin{array}{c}\log \mathrm{FC} \\
(\mathrm{L} 1 \text { vs. S1) }\end{array}$ & Chromosome & $\begin{array}{c}\text { Mirbase } \\
\text { accession no. }\end{array}$ \\
\hline \multirow[t]{51}{*}{ Up-regulated } & hsa-let-7c & 2.574462 & 1.3642709 & $\operatorname{chr} 21$ & MIMAT0000064 \\
\hline & hsa-let-7g-5p & 2.0787826 & 1.0557389 & $\operatorname{chr} 3$ & MIMAT0000414 \\
\hline & hsa-miR-1 & 6.5372915 & 2.708693 & $\operatorname{chr} 18$ & MIMAT0000416 \\
\hline & hsa-miR-101-3p & 7.129267 & 2.8337538 & chrl & MIMAT0000099 \\
\hline & hsa-miR-127-3p & 2.284758 & 1.1920414 & chr14 & MIMAT0000446 \\
\hline & hsa-miR-1306-3p & 2.2638326 & 1.1787672 & $\operatorname{chr} 22$ & MIMAT0005950 \\
\hline & hsa-miR-133b & 38.066643 & 5.2504554 & chr6 & MIMAT0000770 \\
\hline & hsa-miR-144-5p & 7.3764052 & 2.882918 & $\operatorname{chr} 17$ & MIMAT0004600 \\
\hline & hsa-miR-152 & 7.540236 & 2.9146097 & $\operatorname{chr17}$ & MIMAT0000438 \\
\hline & hsa-miR-154-3p & 6.04454 & 2.5956326 & chr14 & MIMAT0000453 \\
\hline & hsa-miR-154-5p & 3.067223 & 1.6169331 & chr14 & MIMAT0000452 \\
\hline & hsa-miR-155-5p & 7.5116105 & 2.9091222 & $\operatorname{chr} 21$ & MIMAT0000646 \\
\hline & hsa-miR-181c-5p & 5.300414 & 2.406105 & chr19 & MIMAT0000258 \\
\hline & hsa-miR-193a-3p & 31.360935 & 4.9708967 & $\operatorname{chr17}$ & MIMAT0000459 \\
\hline & hsa-miR-194-5p & 6.75868 & 2.7567415 & chr1 & MIMAT0000460 \\
\hline & hsa-miR-195-5p & 2.3303063 & 1.2205195 & $\operatorname{chr} 17$ & MIMAT0000461 \\
\hline & hsa-miR-1973 & 7.752263 & 2.9546175 & $\operatorname{chr} 4$ & MIMAT0009448 \\
\hline & hsa-miR-199a-3p & 2.136898 & 1.0955181 & chrl & MIMAT0000232 \\
\hline & hsa-miR-199b-5p & 2.0532408 & 1.0379028 & $\operatorname{chr} 9$ & MIMAT0000263 \\
\hline & hsa-miR-202-3p & 37.374104 & 5.223967 & chr10 & MIMAT0002811 \\
\hline & hsa-miR-214-3p & 2.0166378 & 1.0119519 & chrl & MIMAT0000271 \\
\hline & hsa-miR-218-5p & 6.466407 & 2.6929643 & $\operatorname{chr} 4$ & MIMAT0000275 \\
\hline & hsa-miR-221-3p & 2.6082892 & 1.3831038 & $\operatorname{chrX}$ & MIMAT0000278 \\
\hline & hsa-miR-222-3p & 3.7729478 & 1.9156921 & $\operatorname{chrX}$ & MIMAT0000279 \\
\hline & hsa-miR-28-5p & 2.027596 & 1.0197701 & $\operatorname{chr} 3$ & MIMAT0000085 \\
\hline & hsa-miR-299-3p & 28.977842 & 4.8568783 & chr14 & MIMAT0000687 \\
\hline & hsa-miR-299-5p & 2.3908083 & 1.2574985 & chr14 & MIMAT0002890 \\
\hline & hsa-miR-30e-3p & 5.7678924 & 2.5280442 & chrl & MIMAT0000693 \\
\hline & hsa-miR-3125 & 7.3044753 & 2.8687806 & $\operatorname{chr} 2$ & MIMAT0014988 \\
\hline & hsa-miR-3127-5p & 7.3825746 & 2.884124 & $\operatorname{chr} 2$ & MIMAT0014990 \\
\hline & hsa-miR-323a-3p & 25.762617 & 4.687207 & chr14 & MIMAT0000755 \\
\hline & hsa-miR-33b-3p & 6.059397 & 2.5991743 & $\operatorname{chr17}$ & MIMAT0004811 \\
\hline & hsa-miR-342-3p & 2.086935 & 1.0613856 & chr14 & MIMAT0000753 \\
\hline & hsa-miR-361-3p & 7.1666164 & 2.8412921 & $\operatorname{chrX}$ & MIMAT0004682 \\
\hline & hsa-miR-362-5p & 3.0538094 & 1.61061 & $\operatorname{chrX}$ & MIMAT0000705 \\
\hline & hsa-miR-3620-5p & 88.913635 & 6.474333 & chr1 & MIMAT0022967 \\
\hline & hsa-miR-3622b-5p & 6.9455557 & 2.7960901 & chr8 & MIMAT0018005 \\
\hline & hsa-miR-363-3p & 5.4890895 & 2.4565668 & $\operatorname{chrX}$ & MIMAT0000707 \\
\hline & hsa-miR-3682-3p & 96.9888 & 6.599746 & $\operatorname{chr} 2$ & MIMAT0018110 \\
\hline & hsa-miR-370 & 6.1665797 & 2.6244705 & chr14 & MIMAT0000722 \\
\hline & hsa-miR-376c-3p & 2.002838 & 1.0020456 & chr14 & MIMAT0000720 \\
\hline & hsa-miR-379-5p & 107.84705 & 6.752843 & chr14 & MIMAT0000733 \\
\hline & hsa-miR-381-3p & 2.1550357 & 1.1077118 & $\operatorname{chr14}$ & MIMAT0000736 \\
\hline & hsa-miR-382-5p & 2.920635 & 1.546282 & chr14 & MIMAT0000737 \\
\hline & hsa-miR-3917 & 6.9297132 & 2.7927957 & chrl & MIMAT0018191 \\
\hline & hsa-miR-3923 & 178.99112 & 7.483744 & chr3 & MIMAT0018198 \\
\hline & hsa-miR-409-3p & 4.9062624 & 2.2946243 & chr14 & MIMAT0001639 \\
\hline & hsa-miR-411-5p & 7.6058702 & 2.9271133 & chr14 & MIMAT0003329 \\
\hline & hsa-miR-4476 & 41.244473 & 5.366129 & chr9 & MIMAT0019003 \\
\hline & hsa-miR-4535 & 80.93671 & 6.338722 & $\operatorname{chr} 22$ & MIMAT0019075 \\
\hline & hsa-miR-4539 & 91.96671 & 6.52304 & $\operatorname{chr} 14$ & MIMAT0019082 \\
\hline
\end{tabular}


Table III. Continued.

\begin{tabular}{|c|c|c|c|c|c|}
\hline Regulation & $\begin{array}{c}\text { Systematic } \\
\text { name }\end{array}$ & $\begin{array}{c}\mathrm{FC} \\
(\mathrm{L} 1 \text { vs. S1) }\end{array}$ & $\begin{array}{c}\text { Log FC } \\
(\text { L1 vs. S1) }\end{array}$ & Chromosome & $\begin{array}{c}\text { Mirbase } \\
\text { accession no. }\end{array}$ \\
\hline & hsa-miR-4632-5p & 2.642979 & 1.4021649 & chr1 & MIMAT0022977 \\
\hline & hsa-miR-4698 & 110.092384 & 6.782571 & $\operatorname{chr} 12$ & MIMAT0019793 \\
\hline & hsa-miR-4716-3p & 2.1197543 & 1.0838971 & $\operatorname{chr} 15$ & MIMAT0019827 \\
\hline & hsa-miR-4740-5p & 7.15094 & 2.8381329 & $\operatorname{chr17}$ & MIMAT0019869 \\
\hline & hsa-miR-4743-5p & 41.097565 & 5.360981 & $\operatorname{chr} 18$ & MIMAT0019874 \\
\hline & hsa-miR-4749-3p & 7.212792 & 2.8505578 & $\operatorname{chr19}$ & MIMAT0019886 \\
\hline & hsa-miR-4750-5p & 7.316306 & 2.8711154 & $\operatorname{chr19}$ & MIMAT0019887 \\
\hline & hsa-miR-4754 & 2.11079 & 1.0777831 & chr19 & MIMAT0019894 \\
\hline & hsa-miR-487a & 32.68032 & 5.03035 & chr14 & MIMAT0002178 \\
\hline & hsa-miR-487b & 2.2653325 & 1.1797228 & chr14 & MIMAT0003180 \\
\hline & hsa-miR-489 & 7.1415405 & 2.8362353 & $\operatorname{chr} 7$ & MIMAT0002805 \\
\hline & hsa-miR-493-5p & 2.8272614 & 1.4994053 & chr14 & MIMAT0002813 \\
\hline & hsa-miR-495-3p & 2.1915793 & 1.1319709 & chr14 & MIMAT0002817 \\
\hline & hsa-miR-5003-3p & 74.188484 & 6.2131233 & $\operatorname{chr} 5$ & MIMAT0021026 \\
\hline & hsa-miR-500a-3p & 5.8292727 & 2.543316 & $\operatorname{chrX}$ & MIMAT0002871 \\
\hline & hsa-miR-502-3p & 30.460754 & 4.9288797 & $\operatorname{chrX}$ & MIMAT0004775 \\
\hline & hsa-miR-5096 & 5.691079 & 2.5087023 & $\operatorname{chr} 4$ & MIMAT0020603 \\
\hline & hsa-miR-513b & 2.4948008 & 1.3189247 & $\operatorname{chrX}$ & MIMAT0005788 \\
\hline & hsa-miR-5189 & 7.34395 & 2.8765562 & chr16 & MIMAT0021120 \\
\hline & hsa-miR-532-3p & 30.798903 & 4.944807 & $\operatorname{chrX}$ & MIMAT0004780 \\
\hline & hsa-miR-539-5p & 7.7136526 & 2.9474142 & chr14 & MIMAT0003163 \\
\hline & hsa-miR-543 & 37.697845 & 5.23641 & chr14 & MIMAT0004954 \\
\hline & hsa-miR-5581-5p & 2.7262392 & 1.4469122 & chrl & MIMAT0022275 \\
\hline & hsa-miR-584-5p & 39.059162 & 5.287589 & $\operatorname{chr} 5$ & MIMAT0003249 \\
\hline & hsa-miR-6075 & 7.67851 & 2.9408264 & $\operatorname{chr} 5$ & MIMAT0023700 \\
\hline & hsa-miR-6132 & 7.5204616 & 2.9108212 & $\operatorname{chr} 7$ & MIMAT0024616 \\
\hline & hsa-miR-6508-5p & 6.424311 & 2.6835418 & $\operatorname{chr} 21$ & MIMAT0025472 \\
\hline & hsa-miR-6512-5p & 29.820745 & 4.8982444 & $\operatorname{chr} 2$ & MIMAT0025480 \\
\hline & hsa-miR-652-3p & 7.47874 & 2.9027953 & $\operatorname{chrX}$ & MIMAT0003322 \\
\hline & hsa-miR-654-3p & 2.6468177 & 1.4042588 & chr14 & MIMAT0004814 \\
\hline & hsa-miR-660-5p & 2.1465678 & 1.1020317 & $\operatorname{chrX}$ & MIMAT0003338 \\
\hline & hsa-miR-718 & 6.8719115 & 2.7807114 & $\operatorname{chrX}$ & MIMAT0012735 \\
\hline & hsa-miR-887 & 33.127758 & 5.0499687 & $\operatorname{chr} 5$ & MIMAT0004951 \\
\hline \multirow{18}{*}{$\begin{array}{l}\text { Down- } \\
\text { regulated }\end{array}$} & hsa-miR-1225-3p & -5.8417506 & -2.5464008 & chr16 & MIMAT0005573 \\
\hline & hsa-miR-1238-3p & -8.677957 & -3.1173553 & chr19 & MIMAT0005593 \\
\hline & hsa-miR-126-5p & -4.150803 & -2.0533905 & $\operatorname{chr} 9$ & MIMAT0000444 \\
\hline & hsa-miR-1273f & -4.8539524 & -2.27916 & chrl & MIMAT0020601 \\
\hline & hsa-miR-141-3p & -2.0020258 & -1.0014606 & $\operatorname{chr12}$ & MIMAT0000432 \\
\hline & hsa-miR-142-3p & -5.4498663 & -2.4462209 & $\operatorname{chr17}$ & MIMAT0000434 \\
\hline & hsa-miR-1469 & -2.2450392 & -1.1667407 & $\operatorname{chr} 15$ & MIMAT0007347 \\
\hline & hsa-miR-193b-3p & -2.9037018 & -1.5378933 & chr16 & MIMAT0002819 \\
\hline & hsa-miR-193b-5p & -12.566032 & -3.6514573 & $\operatorname{chr16}$ & MIMAT0004767 \\
\hline & hsa-miR-1972 & -2.4747171 & -1.3072636 & chr16 & MIMAT0009447 \\
\hline & hsa-miR-19a-3p & -6.396935 & -2.6773808 & $\operatorname{chr13}$ & MIMAT0000073 \\
\hline & hsa-miR-210 & -11.999909 & -3.5849516 & chr11 & MIMAT0000267 \\
\hline & hsa-miR-30b-3p & -4.5346327 & -2.1809857 & $\operatorname{chr} 8$ & MIMAT0004589 \\
\hline & hsa-miR-3138 & -4.882965 & -2.2877574 & $\operatorname{chr} 4$ & MIMAT0015006 \\
\hline & hsa-miR-335-3p & -4.2380176 & -2.0833895 & $\operatorname{chr} 7$ & MIMAT0004703 \\
\hline & hsa-miR-338-5p & -3.542421 & -1.8247358 & $\operatorname{chr17}$ & MIMAT0004701 \\
\hline & hsa-miR-3653 & -3.0687642 & -1.6176578 & $\operatorname{chr} 22$ & MIMAT0018073 \\
\hline & hsa-miR-3679-3p & -4.8174143 & -2.268259 & chr2 & MIMAT0018105 \\
\hline
\end{tabular}


Table III. Continued.

\begin{tabular}{|c|c|c|c|c|c|}
\hline Regulation & $\begin{array}{c}\text { Systematic } \\
\text { name }\end{array}$ & $\begin{array}{c}\text { FC } \\
(\mathrm{L} 1 \text { vs. S1) }\end{array}$ & $\begin{array}{c}\log \mathrm{FC} \\
(\mathrm{L} 1 \text { vs. S1) }\end{array}$ & Chromosome & $\begin{array}{c}\text { Mirbase } \\
\text { accession no. }\end{array}$ \\
\hline & hsa-miR-372 & -3.2183118 & -1.6863041 & chr19 & MIMAT0000724 \\
\hline & hsa-miR-373-3p & -4.5374827 & -2.1818922 & chr19 & MIMAT0000726 \\
\hline & hsa-miR-3907 & -4.354147 & -2.12239 & $\operatorname{chr} 7$ & MIMAT0018179 \\
\hline & hsa-miR-4287 & -12.793394 & -3.6773272 & chr8 & MIMAT0016917 \\
\hline & hsa-miR-4324 & -2.4016316 & -1.2640148 & chr19 & MIMAT0016876 \\
\hline & hsa-miR-4429 & -4.713071 & -2.2366674 & $\operatorname{chr} 2$ & MIMAT0018944 \\
\hline & hsa-miR-4472 & -2.5906193 & -1.373297 & $\operatorname{chr} 12$ & MIMAT0018999 \\
\hline & hsa-miR-4484 & -5.330914 & -2.414383 & $\operatorname{chr} 10$ & MIMAT0019018 \\
\hline & hsa-miR-4486 & -2.6245956 & -1.3920952 & chr11 & MIMAT0019020 \\
\hline & hsa-miR-4649-3p & -35.55725 & -5.152072 & $\operatorname{chr} 7$ & MIMAT0019712 \\
\hline & hsa-miR-4767 & -2.7031322 & -1.4346321 & $\operatorname{chrX}$ & MIMAT0019919 \\
\hline & hsa-miR-4783-3p & -2.6541424 & -1.4082458 & chr2 & MIMAT0019947 \\
\hline & hsa-miR-4800-5p & -2.005754 & -1.0041447 & chr4 & MIMAT0019978 \\
\hline & hsa-miR-514b-5p & -2.4455242 & -1.2901437 & $\operatorname{chrX}$ & MIMAT0015087 \\
\hline & hsa-miR-516a-3p & -15.977234 & -3.9979458 & chr19 & MIMAT0006778 \\
\hline & hsa-miR-518a-5p & -5.789181 & -2.5333593 & chr19 & MIMAT0005457 \\
\hline & hsa-miR-518c-3p & -2.3767946 & -1.2490172 & chr19 & MIMAT0002848 \\
\hline & hsa-miR-520b & -5.798103 & -2.5355809 & chr19 & MIMAT0002843 \\
\hline & hsa-miR-523-3p & -2.0523486 & -1.0372758 & chr19 & MIMAT0002840 \\
\hline & hsa-miR-5585-3p & -4.71074 & -2.2359538 & chr1 & MIMAT0022286 \\
\hline & hsa-miR-590-5p & -3.056939 & -1.6120877 & $\operatorname{chr} 7$ & MIMAT0003258 \\
\hline & hsa-miR-623 & -2.361158 & -1.2394946 & $\operatorname{chr13}$ & MIMAT0003292 \\
\hline & hsa-miR-659-3p & -4.1545143 & -2.0546799 & $\operatorname{chr} 22$ & MIMAT0003337 \\
\hline & hsa-miR-664b-3p & -2.9298499 & -1.5508268 & $\operatorname{chrX}$ & MIMAT0022272 \\
\hline & hsa-miR-765 & -12.846773 & -3.683334 & chrl & MIMAT0003945 \\
\hline & hsa-miR-766-3p & -2.3770628 & -1.24918 & $\operatorname{chrX}$ & MIMAT0003888 \\
\hline & hsa-miR-770-5p & -2.219985 & -1.1505499 & chr14 & MIMAT0003948 \\
\hline & hsa-miR-877-3p & -3.1021721 & -1.6332787 & chr6 & MIMAT0004950 \\
\hline
\end{tabular}

15 cases with normal MC were used for validation of microarray data.

RNA extraction. About $200 \mathrm{mg}$ of homogenized placenta tissue was used for extraction of total RNA by using TRIzol reagent (Invitrogen, Carlsbad, CA, USA) according to manufacturer's instructions. After quantifying by using Nanodrop spectrophotometer (Nanodrop Technologies, Wilmington, Delaware, USA), extracted RNA was aliquoted and stored at $-80^{\circ} \mathrm{C}$.

miRNAs expression analysis using miRNA array. miRNA profiling was performed using Affymetrix microRNA 4.0 Array (Santa Clara, CA, US), which covering 2,578 human microRNAs annotated in miRBase V2.0. Briefly, $1 \mu \mathrm{g}$ of each sample was labeled with Biotin using the FlashTag ${ }^{\text {TM }}$ Biotin HSR RNA Labeling Kit (Affymetrix) and then hybridized overnight with the array according to the manufacturer's protocols. After washing and staining, the hybridized slides were read by a GeneChip Scanner 3000 7G (Affymetrix). The raw data were exported by GeneChip Command Console Software Version 4.0 (Affymetrix). The microarray data have been deposited in NCBI's

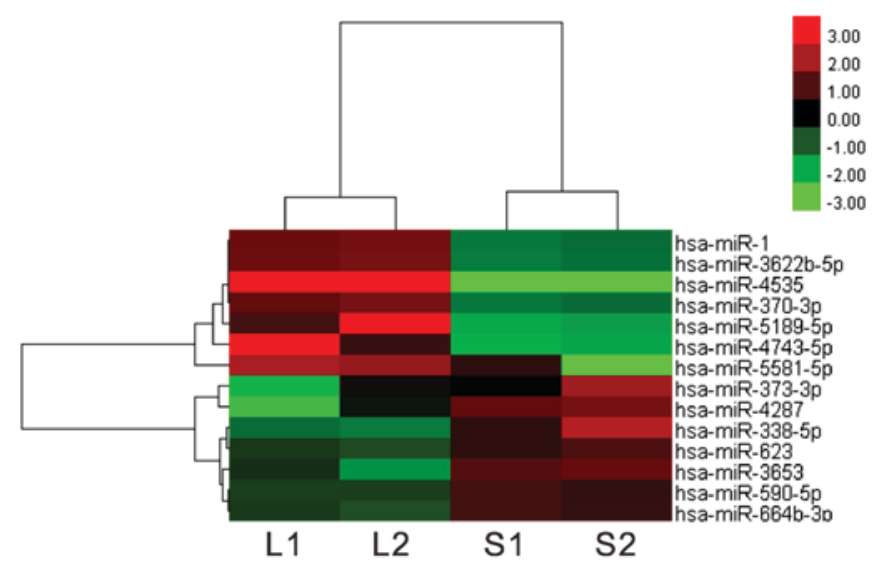

Figure 2. The 14 DEMs differentiated larger twins (L1 and L2) from smaller twins (S1 and S2). The hierarchical clustering analysis was shown.

Gene Expression Omnibus database (GEO, http://www.ncbi. nlm.nih. gov/geo) under accession number GSE98146. miRNAs exhibited Fold Change $>=2.0$ and P-value $<0.05$ were identified as significant differentially expressed miRNAs (DEMs). miRNA 
Table IV. DEMs (L2 vs. S2).

\begin{tabular}{|c|c|c|c|c|c|}
\hline Regulation & $\begin{array}{c}\text { Systematic } \\
\text { name }\end{array}$ & $\begin{array}{c}\text { FC } \\
(\mathrm{L} 1 \text { vs. S1) }\end{array}$ & $\begin{array}{c}\log \mathrm{FC} \\
(\mathrm{L} 1 \text { vs. S1) }\end{array}$ & Chromosome & $\begin{array}{c}\text { Mirbase } \\
\text { accession no. }\end{array}$ \\
\hline \multirow[t]{51}{*}{ Up-regulated } & hsa-miR-1 & 6.2799373 & 2.6507502 & $\operatorname{chr} 18$ & MIMAT0000416 \\
\hline & hsa-miR-101-3p & 6.4381766 & 2.6866522 & chr1 & MIMAT0000099 \\
\hline & hsa-miR-1236-5p & 6.4806085 & 2.6961293 & chr6 & MIMAT0022945 \\
\hline & hsa-miR-1238-3p & 6.655472 & 2.734541 & chr19 & MIMAT0005593 \\
\hline & hsa-miR-1290 & 152.34323 & 7.2511816 & chrl & MIMAT0005880 \\
\hline & hsa-miR-133b & 37.049896 & 5.2113976 & chr6 & MIMAT0000770 \\
\hline & hsa-miR-135b-5p & 94.51581 & 6.562484 & chrl & MIMAT0000758 \\
\hline & hsa-miR-136-3p & 29.46167 & 4.8807673 & $\operatorname{chr} 14$ & MIMAT0004606 \\
\hline & hsa-miR-136-5p & 6.712153 & 2.7467756 & chr14 & MIMAT0000448 \\
\hline & hsa-miR-139-3p & 6.2614946 & 2.646507 & chr11 & MIMAT0004552 \\
\hline & hsa-miR-1469 & 26.883722 & 4.748661 & $\operatorname{chr} 15$ & MIMAT0007347 \\
\hline & hsa-miR-149-3p & 5.559239 & 2.4748874 & chr2 & MIMAT0004609 \\
\hline & hsa-miR-154-3p & 80.835304 & 6.3369136 & chr14 & MIMAT0000453 \\
\hline & hsa-miR-184 & 107.06464 & 6.742338 & $\operatorname{chr} 15$ & MIMAT0000454 \\
\hline & hsa-miR-191-3p & 5.199818 & 2.3784611 & $\operatorname{chr} 3$ & MIMAT0001618 \\
\hline & hsa-miR-193a-3p & 30.860113 & 4.9476714 & $\operatorname{chr17}$ & MIMAT0000459 \\
\hline & hsa-miR-193b-5p & 6.7797785 & 2.761238 & chr16 & MIMAT0004767 \\
\hline & hsa-miR-1972 & 31.809767 & 4.991398 & chr16 & MIMAT0009447 \\
\hline & hsa-miR-198 & 7.100607 & 2.8279424 & $\operatorname{chr} 3$ & MIMAT0000228 \\
\hline & hsa-miR-19a-3p & 6.9222608 & 2.7912433 & $\operatorname{chr13}$ & MIMAT0000073 \\
\hline & hsa-miR-204-5p & 29.051617 & 4.8605466 & $\operatorname{chr} 9$ & MIMAT0000265 \\
\hline & hsa-miR-2114-5p & 128.1817 & 7.0020466 & $\operatorname{chrX}$ & MIMAT0011156 \\
\hline & hsa-miR-218-5p & 6.376806 & 2.672834 & $\operatorname{chr} 4$ & MIMAT0000275 \\
\hline & hsa-miR-298 & 6.9036517 & 2.7873597 & $\operatorname{chr} 20$ & MIMAT0004901 \\
\hline & hsa-miR-299-3p & 30.239016 & 4.9183393 & $\operatorname{chr14}$ & MIMAT0000687 \\
\hline & hsa-miR-301a-3p & 23.225285 & 4.5376244 & $\operatorname{chr17}$ & MIMAT0000688 \\
\hline & hsa-miR-3127-5p & 6.460783 & 2.691709 & $\operatorname{chr} 2$ & MIMAT0014990 \\
\hline & hsa-miR-3135b & 123.808846 & 6.9519706 & chr6 & MIMAT0018985 \\
\hline & hsa-miR-3147 & 6.529907 & 2.7070625 & $\operatorname{chr} 7$ & MIMAT0015019 \\
\hline & hsa-miR-3173-3p & 166.4288 & 7.3787613 & chr14 & MIMAT0015048 \\
\hline & hsa-miR-3180-3p & 156.06705 & 7.286022 & chr16 & MIMAT0015058 \\
\hline & hsa-miR-3194-5p & 106.03497 & 6.7283964 & $\operatorname{chr} 20$ & MIMAT0015078 \\
\hline & hsa-miR-33b-3p & 5.4492188 & 2.4460495 & $\operatorname{chr17}$ & MIMAT0004811 \\
\hline & hsa-miR-34b-5p & 6.9539423 & 2.797831 & chr11 & MIMAT0000685 \\
\hline & hsa-miR-3610 & 7.0247335 & 2.8124435 & chr8 & MIMAT0017987 \\
\hline & hsa-miR-3620-5p & 119.47107 & 6.9005175 & chrl & MIMAT0022967 \\
\hline & hsa-miR-3622b-5p & 6.797605 & 2.7650266 & chr8 & MIMAT0018005 \\
\hline & hsa-miR-3675-3p & 5.4626126 & 2.4495912 & chr1 & MIMAT0018099 \\
\hline & hsa-miR-370 & 6.585063 & 2.7191973 & chr14 & MIMAT0000722 \\
\hline & hsa-miR-3911 & 136.38383 & 7.091529 & chr9 & MIMAT0018185 \\
\hline & hsa-miR-411-5p & 6.842667 & 2.7745588 & chr14 & MIMAT0003329 \\
\hline & hsa-miR-4252 & 6.649911 & 2.733335 & chrl & MIMAT0016886 \\
\hline & hsa-miR-4257 & 29.410418 & 4.8782554 & chrl & MIMAT0016878 \\
\hline & hsa-miR-4274 & 6.556329 & 2.7128882 & $\operatorname{chr} 4$ & MIMAT0016906 \\
\hline & hsa-miR-4280 & 6.1641645 & 2.6239054 & $\operatorname{chr} 5$ & MIMAT0016911 \\
\hline & hsa-miR-4314 & 6.209586 & 2.6344972 & $\operatorname{chr17}$ & MIMAT0016868 \\
\hline & hsa-miR-4317 & 29.65745 & 4.8903227 & $\operatorname{chr} 18$ & MIMAT0016872 \\
\hline & hsa-miR-4322 & 114.98171 & 6.8452606 & $\operatorname{chr19}$ & MIMAT0016873 \\
\hline & hsa-miR-4327 & 168.50354 & 7.396635 & $\operatorname{chr} 21$ & MIMAT0016889 \\
\hline & hsa-miR-4428 & 6.98875 & 2.8050344 & chr1 & MIMAT0018943 \\
\hline & hsa-miR-4443 & 2.4926476 & 1.3176789 & chr3 & MIMAT0018961 \\
\hline
\end{tabular}


Table IV. Continued.

\begin{tabular}{|c|c|c|c|c|c|}
\hline Regulation & $\begin{array}{c}\text { Systematic } \\
\text { name }\end{array}$ & $\begin{array}{c}\text { FC } \\
(\mathrm{L} 1 \text { vs. S1) }\end{array}$ & $\begin{array}{c}\text { Log FC } \\
(\mathrm{L} 1 \text { vs. S1) }\end{array}$ & Chromosome & $\begin{array}{c}\text { Mirbase } \\
\text { accession no. }\end{array}$ \\
\hline & hsa-miR-4476 & 6.292596 & 2.6536553 & chr9 & MIMAT0019003 \\
\hline & hsa-miR-4482-3p & 6.502412 & 2.700975 & chr10 & MIMAT0020958 \\
\hline & hsa-miR-4484 & 91.229645 & 6.5114307 & chr10 & MIMAT0019018 \\
\hline & hsa-miR-4486 & 37.18235 & 5.216546 & chr11 & MIMAT0019020 \\
\hline & hsa-miR-4496 & 5.89344 & 2.55911 & $\operatorname{chr} 12$ & MIMAT0019031 \\
\hline & hsa-miR-4513 & 131.67114 & 7.0407953 & $\operatorname{chr} 15$ & MIMAT0019050 \\
\hline & hsa-miR-4522 & 111.41926 & 6.7998548 & $\operatorname{chr} 17$ & MIMAT0019060 \\
\hline & hsa-miR-4535 & 84.8799 & 6.407351 & $\operatorname{chr} 22$ & MIMAT0019075 \\
\hline & hsa-miR-4539 & 156.92206 & 7.2939043 & $\operatorname{chr} 14$ & MIMAT0019082 \\
\hline & hsa-miR-4632-5p & 6.771799 & 2.7595391 & chrl & MIMAT0022977 \\
\hline & hsa-miR-4646-5p & 80.15663 & 6.32475 & $\operatorname{chr} 6$ & MIMAT0019707 \\
\hline & hsa-miR-4656 & 112.753174 & 6.817024 & $\operatorname{chr} 7$ & MIMAT0019723 \\
\hline & hsa-miR-4690-5p & 26.123838 & 4.707295 & chr11 & MIMAT0019779 \\
\hline & hsa-miR-4698 & 122.851776 & 6.940775 & $\operatorname{chr} 12$ & MIMAT0019793 \\
\hline & hsa-miR-4734 & 2.1158128 & 1.081212 & $\operatorname{chr} 17$ & MIMAT0019859 \\
\hline & hsa-miR-4740-5p & 140.27261 & 7.1320896 & $\operatorname{chr} 17$ & MIMAT0019869 \\
\hline & hsa-miR-4743-5p & 6.4767523 & 2.6952705 & $\operatorname{chr} 18$ & MIMAT0019874 \\
\hline & hsa-miR-4749-3p & 6.195103 & 2.6311283 & $\operatorname{chr} 19$ & MIMAT0019886 \\
\hline & hsa-miR-4758-5p & 72.96534 & 6.1891394 & $\operatorname{chr} 20$ & MIMAT0019903 \\
\hline & hsa-miR-4767 & 38.017128 & 5.2485776 & $\operatorname{chrX}$ & MIMAT0019919 \\
\hline & hsa-miR-487a & 34.672806 & 5.1157327 & $\operatorname{chr} 14$ & MIMAT0002178 \\
\hline & hsa-miR-5003-3p & 99.04469 & 6.6300077 & $\operatorname{chr} 5$ & MIMAT0021026 \\
\hline & hsa-miR-502-3p & 6.4271126 & 2.6841707 & $\operatorname{chrX}$ & MIMAT0004775 \\
\hline & hsa-miR-5096 & 28.428453 & 4.8292637 & $\operatorname{chr} 4$ & MIMAT0020603 \\
\hline & hsa-miR-513a-5p & 92.79643 & 6.5359974 & $\operatorname{chrX}$ & MIMAT0002877 \\
\hline & hsa-miR-513b & 5.130571 & 2.3591194 & $\operatorname{chrX}$ & MIMAT0005788 \\
\hline & hsa-miR-513c-5p & 5.255153 & 2.3937328 & $\operatorname{chrX}$ & MIMAT0005789 \\
\hline & hsa-miR-514b-5p & 86.46717 & 6.4340806 & $\operatorname{chrX}$ & MIMAT0015087 \\
\hline & hsa-miR-5189 & 28.272223 & 4.8213134 & chr16 & MIMAT0021120 \\
\hline & hsa-miR-518a-5p & 27.765553 & 4.795224 & $\operatorname{chr} 19$ & MIMAT0005457 \\
\hline & hsa-miR-5195-5p & 6.754108 & 2.7557652 & chr14 & MIMAT0021126 \\
\hline & hsa-miR-520b & 6.59573 & 2.7215323 & $\operatorname{chr} 19$ & MIMAT0002843 \\
\hline & hsa-miR-532-3p & 6.8659596 & 2.7794614 & $\operatorname{chrX}$ & MIMAT0004780 \\
\hline & hsa-miR-539-5p & 28.060043 & 4.8104453 & $\operatorname{chr} 14$ & MIMAT0003163 \\
\hline & hsa-miR-543 & 36.802776 & 5.2017426 & chr14 & MIMAT0004954 \\
\hline & hsa-miR-557 & 87.43645 & 6.450163 & chrl & MIMAT0003221 \\
\hline & hsa-miR-5581-5p & 69.77128 & 6.1245613 & chr1 & MIMAT0022275 \\
\hline & hsa-miR-601 & 2.1609044 & 1.1116352 & $\operatorname{chr} 9$ & MIMAT0003269 \\
\hline & hsa-miR-602 & 5.791587 & 2.5339587 & $\operatorname{chr} 9$ & MIMAT0003270 \\
\hline & hsa-miR-605 & 12.47016 & 3.640408 & $\operatorname{chr10}$ & MIMAT0003273 \\
\hline & hsa-miR-6075 & 35.023468 & 5.13025 & $\operatorname{chr} 5$ & MIMAT0023700 \\
\hline & hsa-miR-6081 & 6.4466505 & 2.6885498 & $\operatorname{chr} 9$ & MIMAT0023706 \\
\hline & hsa-miR-6086 & 107.75808 & 6.7516522 & $\operatorname{chrX}$ & MIMAT0023711 \\
\hline & hsa-miR-6087 & 2.2509918 & 1.1705608 & $\operatorname{chrX}$ & MIMAT0023712 \\
\hline & hsa-miR-610 & 150.35155 & 7.232196 & chr11 & MIMAT0003278 \\
\hline & hsa-miR-622 & 5.405943 & 2.4345462 & $\operatorname{chr13}$ & MIMAT0003291 \\
\hline & hsa-miR-630 & 2.1389875 & 1.0969281 & $\operatorname{chr} 15$ & MIMAT0003299 \\
\hline & hsa-miR-6511b-5p & 6.7421665 & 2.7532122 & chr16 & MIMAT0025847 \\
\hline & hsa-miR-659-3p & 68.05403 & 6.0886087 & $\operatorname{chr} 22$ & MIMAT0003337 \\
\hline & hsa-miR-671-5p & 116.28812 & 6.86156 & $\operatorname{chr} 7$ & MIMAT0003880 \\
\hline & hsa-miR-6722-3p & 35.948414 & 5.167856 & $\operatorname{chr} 9$ & MIMAT0025854 \\
\hline
\end{tabular}


Table IV. Continued.

\begin{tabular}{|c|c|c|c|c|c|}
\hline Regulation & $\begin{array}{c}\text { Systematic } \\
\text { name }\end{array}$ & $\begin{array}{c}\text { FC } \\
(\mathrm{L} 1 \text { vs. S1) }\end{array}$ & $\begin{array}{c}\log \mathrm{FC} \\
(\mathrm{L} 1 \text { vs. S1) }\end{array}$ & Chromosome & $\begin{array}{c}\text { Mirbase } \\
\text { accession no. }\end{array}$ \\
\hline \multirow{46}{*}{$\begin{array}{l}\text { Down- } \\
\text { regulated }\end{array}$} & hsa-miR-758-3p & 4.839683 & 2.2749126 & $\operatorname{chr} 14$ & MIMAT0003879 \\
\hline & hsa-miR-765 & 6.2472134 & 2.6432128 & chr1 & MIMAT0003945 \\
\hline & hsa-miR-769-3p & 66.88174 & 6.0635405 & $\operatorname{chr19}$ & MIMAT0003887 \\
\hline & hsa-miR-877-3p & 34.31749 & 5.100872 & chr6 & MIMAT0004950 \\
\hline & hsa-miR-887 & 6.620651 & 2.726973 & $\operatorname{chr} 5$ & MIMAT0004951 \\
\hline & hsa-miR-10a-5p & -3.7419279 & -1.9037818 & $\operatorname{chr} 17$ & MIMAT0000253 \\
\hline & hsa-miR-1281 & -4.1730103 & -2.0610886 & $\operatorname{chr} 22$ & MIMAT0005939 \\
\hline & hsa-miR-1306-3p & -6.1992292 & -2.632089 & $\operatorname{chr} 22$ & MIMAT0005950 \\
\hline & hsa-miR-138-2-3p & -3.1503472 & -1.6555109 & $\operatorname{chr16}$ & MIMAT0004596 \\
\hline & hsa-miR-144-3p & -214.5132 & -7.7449226 & $\operatorname{chr} 17$ & MIMAT0000436 \\
\hline & hsa-miR-148b-3p & -2.2464561 & -1.1676509 & $\operatorname{chr} 12$ & MIMAT0000759 \\
\hline & hsa-miR-150-5p & -3.811245 & -1.9302623 & $\operatorname{chr} 19$ & MIMAT0000451 \\
\hline & hsa-miR-151a-3p & -2.9817586 & -1.5761634 & chr8 & MIMAT0000757 \\
\hline & hsa-miR-197-3p & -3.9836307 & -1.9940839 & chrl & MIMAT0000227 \\
\hline & hsa-miR-3064-5p & -2.292184 & -1.196723 & $\operatorname{chr17}$ & MIMAT0019864 \\
\hline & hsa-miR-3162-3p & -12.083851 & -3.5950084 & chr11 & MIMAT0019213 \\
\hline & hsa-miR-335-3p & -13.996734 & -3.8070183 & $\operatorname{chr} 7$ & MIMAT0004703 \\
\hline & hsa-miR-338-5p & -11.968582 & -3.5811803 & $\operatorname{chr17}$ & MIMAT0004701 \\
\hline & hsa-miR-363-3p & -2.986565 & -1.5784872 & $\operatorname{chrX}$ & MIMAT0000707 \\
\hline & hsa-miR-3651 & -2.0020404 & -1.001471 & $\operatorname{chr} 9$ & MIMAT0018071 \\
\hline & hsa-miR-3653 & -7.8723674 & -2.9767976 & $\operatorname{chr} 22$ & MIMAT0018073 \\
\hline & hsa-miR-3679-3p & -3.019187 & -1.5941601 & $\operatorname{chr} 2$ & MIMAT0018105 \\
\hline & hsa-miR-373-3p & -3.2492068 & -1.7000875 & $\operatorname{chr19}$ & MIMAT0000726 \\
\hline & hsa-miR-378i & -6.4152656 & -2.681509 & $\operatorname{chr} 22$ & MIMAT0019074 \\
\hline & hsa-miR-3923 & -144.07567 & -7.170683 & chr3 & MIMAT0018198 \\
\hline & hsa-miR-4287 & -2.9859235 & -1.5781772 & chr8 & MIMAT0016917 \\
\hline & hsa-miR-4324 & -3.4396935 & -1.78228 & $\operatorname{chr} 19$ & MIMAT0016876 \\
\hline & hsa-miR-4455 & -2.6491299 & -1.4055185 & $\operatorname{chr} 4$ & MIMAT0018977 \\
\hline & hsa-miR-4472 & -3.141099 & -1.6512694 & $\operatorname{chr} 12$ & MIMAT0018999 \\
\hline & hsa-miR-4481 & -4.197097 & -2.0693917 & $\operatorname{chr} 10$ & MIMAT0019015 \\
\hline & hsa-miR-4485 & -3.3330746 & -1.7368536 & chr11 & MIMAT0019019 \\
\hline & hsa-miR-455-3p & -2.3138413 & -1.21029 & $\operatorname{chr} 9$ & MIMAT0004784 \\
\hline & hsa-miR-4707-5p & -2.0739546 & -1.0523844 & chr14 & MIMAT0019807 \\
\hline & hsa-miR-4710 & -22.40822 & -4.485956 & $\operatorname{chr} 14$ & MIMAT0019815 \\
\hline & hsa-miR-4754 & -3.394706 & -1.7632866 & chr19 & MIMAT0019894 \\
\hline & hsa-miR-491-3p & -80.396675 & -6.329064 & $\operatorname{chr} 9$ & MIMAT0004765 \\
\hline & hsa-miR-5190 & -4.129208 & -2.045865 & $\operatorname{chr} 18$ & MIMAT0021121 \\
\hline & hsa-miR-5196-5p & -2.629623 & -1.394856 & $\operatorname{chr} 19$ & MIMAT0021128 \\
\hline & hsa-miR-574-5p & -2.571706 & -1.3627257 & $\operatorname{chr} 4$ & MIMAT0004795 \\
\hline & hsa-miR-584-5p & -3.136044 & -1.6489458 & $\operatorname{chr} 5$ & MIMAT0003249 \\
\hline & hsa-miR-590-5p & -2.542061 & -1.3459988 & $\operatorname{chr} 7$ & MIMAT0003258 \\
\hline & hsa-miR-623 & -3.541924 & -1.8245332 & $\operatorname{chr} 13$ & MIMAT0003292 \\
\hline & hsa-miR-650 & -12.4346285 & -3.6362915 & $\operatorname{chr} 22$ & MIMAT0003320 \\
\hline & hsa-miR-652-5p & -2.726224 & -1.4469041 & $\operatorname{chrX}$ & MIMAT0022709 \\
\hline & hsa-miR-664b-3p & -3.0773356 & -1.6216818 & $\operatorname{chrX}$ & MIMAT0022272 \\
\hline & hsa-miR-766-3p & -7.31454 & -2.870767 & $\operatorname{chrX}$ & MIMAT0003888 \\
\hline
\end{tabular}

target genes were predicted by miRanda (http://www.microrna. org) (18) and TargetScan (http://www.targetscan.org/) (19).
Pathway analysis. To find out the significant pathway of the differential genes, pathway analysis was performed according 
Table V. DEMs (N1 vs. n1).

\begin{tabular}{|c|c|c|c|c|c|}
\hline Regulation & $\begin{array}{c}\text { Systematic } \\
\text { name }\end{array}$ & $\begin{array}{c}\text { FC } \\
\text { (L1 vs. S1) }\end{array}$ & $\begin{array}{c}\log \mathrm{FC} \\
\text { (L1 vs. S1) }\end{array}$ & Chromosome & $\begin{array}{c}\text { Mirbase } \\
\text { accession no. }\end{array}$ \\
\hline \multirow{50}{*}{ Up-regulated } & hsa-let-7f-1-3p & 4.762908 & 2.2518427 & $\operatorname{chr} 9$ & MIMAT0004486 \\
\hline & hsa-miR-1236-5p & 4.8852377 & 2.2884288 & chr6 & MIMAT0022945 \\
\hline & hsa-miR-1290 & 3.285437 & 1.7160853 & chr1 & MIMAT0005880 \\
\hline & hsa-miR-138-2-3p & 2.8810282 & 1.5265838 & chr16 & MIMAT0004596 \\
\hline & hsa-miR-142-3p & 2.217947 & 1.1492249 & chr17 & MIMAT0000434 \\
\hline & hsa-miR-144-5p & 98.02035 & 6.6150093 & chr17 & MIMAT0004600 \\
\hline & hsa-miR-149-3p & 4.419525 & 2.1438913 & chr2 & MIMAT0004609 \\
\hline & hsa-miR-1914-3p & 2.0559897 & 1.0398331 & $\operatorname{chr} 20$ & MIMAT0007890 \\
\hline & hsa-miR-197-5p & 3.2845297 & 1.7156868 & chrl & MIMAT0022691 \\
\hline & hsa-miR-19a-3p & 2.7125516 & 1.4396505 & $\operatorname{chrl3}$ & MIMAT0000073 \\
\hline & hsa-miR-3138 & 5.336239 & 2.4158232 & $\operatorname{chr} 4$ & MIMAT0015006 \\
\hline & hsa-miR-3156-5p & 2.5390592 & 1.3442941 & chr10 & MIMAT0015030 \\
\hline & hsa-miR-3180-3p & 34.78387 & 5.1203465 & chr16 & MIMAT0015058 \\
\hline & hsa-miR-335-3p & 4.254274 & 2.088913 & $\operatorname{chr} 7$ & MIMAT0004703 \\
\hline & hsa-miR-33b-3p & 5.437156 & 2.4428523 & chr17 & MIMAT0004811 \\
\hline & hsa-miR-3675-3p & 30.885275 & 4.9488473 & chrl & MIMAT0018099 \\
\hline & hsa-miR-3679-3p & 4.8394313 & 2.2748375 & chr2 & MIMAT0018105 \\
\hline & hsa-miR-378i & 5.5282373 & 2.4668195 & $\operatorname{chr} 22$ & MIMAT0019074 \\
\hline & hsa-miR-382-5p & 2.0238345 & 1.0170913 & chr14 & MIMAT0000737 \\
\hline & hsa-miR-4257 & 2.8030276 & 1.4869859 & chrl & MIMAT0016878 \\
\hline & hsa-miR-4299 & 2.322742 & 1.2158289 & chrl1 & MIMAT0016851 \\
\hline & hsa-miR-4324 & 2.5575805 & 1.3547796 & $\operatorname{chr} 19$ & MIMAT0016876 \\
\hline & hsa-miR-4442 & 2.2200553 & 1.1505957 & $\operatorname{chr} 3$ & MIMAT0018960 \\
\hline & hsa-miR-4472 & 2.564959 & 1.3589358 & chr12 & MIMAT0018999 \\
\hline & hsa-miR-4476 & 34.042854 & 5.08928 & chr9 & MIMAT0019003 \\
\hline & hsa-miR-4481 & 2.978939 & 1.5747986 & $\operatorname{chr} 10$ & MIMAT0019015 \\
\hline & hsa-miR-4486 & 2.60555 & 1.381588 & chr11 & MIMAT0019020 \\
\hline & hsa-miR-4497 & 2.0459917 & 1.0328002 & chr12 & MIMAT0019032 \\
\hline & hsa-miR-4505 & 2.1049914 & 1.0738144 & chr14 & MIMAT0019041 \\
\hline & hsa-miR-4513 & 31.151861 & 4.9612465 & chr15 & MIMAT0019050 \\
\hline & hsa-miR-4656 & 2.8565521 & 1.5142748 & $\operatorname{chr} 7$ & MIMAT0019723 \\
\hline & hsa-miR-4698 & 2.3614159 & 1.2396522 & chr12 & MIMAT0019793 \\
\hline & hsa-miR-4731-3p & 3.035372 & 1.6018734 & $\operatorname{chr17}$ & MIMAT0019854 \\
\hline & hsa-miR-4740-5p & 2.4809349 & 1.3108839 & chr17 & MIMAT0019869 \\
\hline & hsa-miR-4746-3p & 2.4210067 & 1.2756071 & chr19 & MIMAT0019881 \\
\hline & hsa-miR-4767 & 3.1487603 & 1.654784 & $\operatorname{chrX}$ & MIMAT0019919 \\
\hline & hsa-miR-4788 & 2.6694448 & 1.4165397 & $\operatorname{chr} 3$ & MIMAT0019958 \\
\hline & hsa-miR-486-5p & 2.3081188 & 1.2067175 & chr8 & MIMAT0002177 \\
\hline & hsa-miR-493-5p & 2.2956579 & 1.1989076 & chr14 & MIMAT0002813 \\
\hline & hsa-miR-514b-5p & 13.624513 & 3.7681327 & $\operatorname{chrX}$ & MIMAT0015087 \\
\hline & hsa-miR-518a-5p & 2.4678388 & 1.3032482 & chr19 & MIMAT0005457 \\
\hline & hsa-miR-520f & 4.3717 & 2.1281943 & chr19 & MIMAT0002830 \\
\hline & hsa-miR-557 & 3.5224607 & 1.8165836 & chrl & MIMAT0003221 \\
\hline & hsa-miR-6087 & 2.4719765 & 1.305665 & $\operatorname{chrX}$ & MIMAT0023712 \\
\hline & hsa-miR-6127 & 2.003823 & 1.0027552 & chrl & MIMAT0024610 \\
\hline & hsa-miR-650 & 3.9777071 & 1.991937 & $\operatorname{chr} 22$ & MIMAT0003320 \\
\hline & hsa-miR-652-5p & 2.5595675 & 1.3559 & $\operatorname{chrX}$ & MIMAT0022709 \\
\hline & hsa-miR-6722-3p & 2.8041553 & 1.4875662 & chr9 & MIMAT0025854 \\
\hline & hsa-miR-769-3p & 42.50052 & 5.4094086 & chr19 & MIMAT0003887 \\
\hline & hsa-miR-887 & 113.96282 & 6.8324194 & $\operatorname{chr} 5$ & MIMAT0004951 \\
\hline
\end{tabular}


Table V. Continued.

\begin{tabular}{|c|c|c|c|c|c|}
\hline Regulation & $\begin{array}{c}\text { Systematic } \\
\text { name }\end{array}$ & $\begin{array}{c}\mathrm{FC} \\
(\mathrm{L} 1 \text { vs. S1) }\end{array}$ & $\begin{array}{c}\log \mathrm{FC} \\
(\mathrm{L} 1 \text { vs. S1) }\end{array}$ & Chromosome & $\begin{array}{c}\text { Mirbase } \\
\text { accession no. }\end{array}$ \\
\hline \multirow{51}{*}{$\begin{array}{l}\text { Down- } \\
\text { regulated }\end{array}$} & hsa-let-7c & -2.015736 & -1.0113068 & $\operatorname{chr} 21$ & MIMAT0000064 \\
\hline & hsa-miR-101-3p & -30.543362 & -4.932787 & chrl & MIMAT0000099 \\
\hline & hsa-miR-1225-3p & -6.48305 & -2.6966727 & chr16 & MIMAT0005573 \\
\hline & hsa-miR-126-5p & -7.1831927 & -2.8446252 & $\operatorname{chr} 9$ & MIMAT0000444 \\
\hline & hsa-miR-1281 & -2.6692638 & -1.4164419 & $\operatorname{chr} 22$ & MIMAT0005939 \\
\hline & hsa-miR-133b & -6.7808595 & -2.7614682 & chr6 & MIMAT0000770 \\
\hline & hsa-miR-136-3p & -32.852886 & -5.037948 & $\operatorname{chr14}$ & MIMAT0004606 \\
\hline & hsa-miR-136-5p & -3.713002 & -1.8925861 & chr14 & MIMAT0000448 \\
\hline & hsa-miR-139-3p & -7.6147995 & -2.928806 & chr11 & MIMAT0004552 \\
\hline & hsa-miR-1469 & -7.4298234 & -2.893328 & $\operatorname{chr} 15$ & MIMAT0007347 \\
\hline & hsa-miR-148a-3p & -2.1474736 & -1.1026404 & $\operatorname{chr} 7$ & MIMAT0000243 \\
\hline & hsa-miR-152 & -7.315202 & -2.8708978 & $\operatorname{chr} 17$ & MIMAT0000438 \\
\hline & hsa-miR-154-3p & -40.05453 & -5.3238935 & chr14 & MIMAT0000453 \\
\hline & hsa-miR-155-5p & -6.8148932 & -2.768691 & $\operatorname{chr} 21$ & MIMAT0000646 \\
\hline & hsa-miR-181c-5p & -7.4166875 & -2.890775 & $\operatorname{chr19}$ & MIMAT0000258 \\
\hline & hsa-miR-183-5p & -7.3213196 & -2.8721037 & chr7 & MIMAT0000261 \\
\hline & hsa-miR-184 & -2.7409573 & -1.4546798 & $\operatorname{chr} 15$ & MIMAT0000454 \\
\hline & hsa-miR-193a-3p & -3.5614202 & -1.8324527 & $\operatorname{chr} 17$ & MIMAT0000459 \\
\hline & hsa-miR-193a-5p & -7.283015 & -2.8645358 & $\operatorname{chr} 17$ & MIMAT0004614 \\
\hline & hsa-miR-1972 & -33.596 & -5.0702176 & chr16 & MIMAT0009447 \\
\hline & hsa-miR-198 & -7.6496506 & -2.9353938 & $\operatorname{chr} 3$ & MIMAT0000228 \\
\hline & hsa-miR-202-3p & -4.0569763 & -2.0204048 & $\operatorname{chr} 10$ & MIMAT0002811 \\
\hline & hsa-miR-2114-5p & -102.90411 & -6.685157 & $\operatorname{chrX}$ & MIMAT0011156 \\
\hline & hsa-miR-218-5p & -6.8879266 & -2.7840698 & chr4 & MIMAT0000275 \\
\hline & hsa-miR-222-3p & -2.7792513 & -1.4746963 & $\operatorname{chrX}$ & MIMAT0000279 \\
\hline & hsa-miR-299-3p & -7.6879406 & -2.9425972 & chr14 & MIMAT0000687 \\
\hline & hsa-miR-301a-3p & -36.397095 & -5.1857514 & $\operatorname{chr} 17$ & MIMAT0000688 \\
\hline & hsa-miR-3064-5p & -40.484715 & -5.3393054 & $\operatorname{chr} 17$ & MIMAT0019864 \\
\hline & hsa-miR-30b-3p & -14.569369 & -3.8648665 & chr8 & MIMAT0004589 \\
\hline & hsa-miR-3125 & -2.843465 & -1.5076501 & $\operatorname{chr} 2$ & MIMAT0014988 \\
\hline & hsa-miR-3127-5p & -2.7899294 & -1.4802287 & $\operatorname{chr} 2$ & MIMAT0014990 \\
\hline & hsa-miR-3135b & -4.5898676 & -2.1984525 & chr6 & MIMAT0018985 \\
\hline & hsa-miR-3147 & -7.3836718 & -2.8843384 & chr7 & MIMAT0015019 \\
\hline & hsa-miR-3173-3p & -3.5254762 & -1.8178182 & chr14 & MIMAT0015048 \\
\hline & hsa-miR-3194-5p & -2.9631052 & -1.5671098 & $\operatorname{chr} 20$ & MIMAT0015078 \\
\hline & hsa-miR-323a-3p & -7.243867 & -2.85676 & $\operatorname{chr14}$ & MIMAT0000755 \\
\hline & hsa-miR-345-3p & -3.170144 & -1.6645484 & chr14 & MIMAT0022698 \\
\hline & hsa-miR-34b-5p & -89.56483 & -6.4848604 & chr11 & MIMAT0000685 \\
\hline & hsa-miR-362-3p & -6.8308253 & -2.77206 & $\operatorname{chr} X$ & MIMAT0004683 \\
\hline & hsa-miR-3620-5p & -3.0599833 & -1.6135237 & chrl & MIMAT0022967 \\
\hline & hsa-miR-3660 & -7.1529465 & -2.8385377 & $\operatorname{chr} 5$ & MIMAT0018081 \\
\hline & hsa-miR-377-3p & -2.1646178 & -1.1141124 & chr14 & MIMAT0000730 \\
\hline & hsa-miR-3907 & -2.053038 & -1.0377603 & $\operatorname{chr} 7$ & MIMAT0018179 \\
\hline & hsa-miR-3923 & -86.46017 & -6.433964 & chr3 & MIMAT0018198 \\
\hline & hsa-miR-411-5p & -7.0822854 & -2.824215 & chr14 & MIMAT0003329 \\
\hline & hsa-miR-4252 & -103.971664 & -6.7000465 & chrl & MIMAT0016886 \\
\hline & hsa-miR-4280 & -6.009121 & -2.587154 & $\operatorname{chr} 5$ & MIMAT0016911 \\
\hline & hsa-miR-4317 & -34.604424 & -5.1128845 & $\operatorname{chr} 18$ & MIMAT0016872 \\
\hline & hsa-miR-4322 & -91.20365 & -6.5110197 & $\operatorname{chr} 19$ & MIMAT0016873 \\
\hline & hsa-miR-4428 & -6.9082146 & -2.788313 & chr1 & MIMAT0018943 \\
\hline & hsa-miR-4522 & -40.902264 & -5.354109 & $\operatorname{chr} 17$ & MIMAT0019060 \\
\hline
\end{tabular}


Table V. Continued.

\begin{tabular}{|c|c|c|c|c|c|}
\hline Regulation & $\begin{array}{l}\text { Systematic } \\
\text { name }\end{array}$ & $\begin{array}{c}\text { FC } \\
\text { (L1 vs. S1) }\end{array}$ & $\begin{array}{l}\log \mathrm{FC} \\
\text { (L1 vs. S1) }\end{array}$ & Chromosome & $\begin{array}{c}\text { Mirbase } \\
\text { accession no. }\end{array}$ \\
\hline & hsa-miR-4539 & -3.3245769 & -1.7331707 & chr14 & MIMAT0019082 \\
\hline & hsa-miR-455-5p & -28.737402 & -4.8448577 & chr9 & MIMAT0003150 \\
\hline & hsa-miR-4632-5p & -7.527243 & -2.9121215 & chrl & MIMAT0022977 \\
\hline & hsa-miR-4646-5p & -2.8159547 & -1.4936241 & chr6 & MIMAT0019707 \\
\hline & hsa-miR-4649-3p & -7.12472 & -2.8328333 & $\operatorname{chr} 7$ & MIMAT0019712 \\
\hline & hsa-miR-4690-5p & -7.212258 & -2.850451 & chr11 & MIMAT0019779 \\
\hline & hsa-miR-4749-3p & -6.8722167 & -2.7807755 & chr19 & MIMAT0019886 \\
\hline & hsa-miR-487a & -35.818806 & -5.1626453 & chr14 & MIMAT0002178 \\
\hline & hsa-miR-489 & -7.070114 & -2.8217335 & $\operatorname{chr} 7$ & MIMAT0002805 \\
\hline & hsa-miR-491-3p & -5.64386 & -2.4966822 & chr9 & MIMAT0004765 \\
\hline & hsa-miR-5003-3p & -7.107153 & -2.8292718 & $\operatorname{chr} 5$ & MIMAT0021026 \\
\hline & hsa-miR-502-3p & -14.068433 & -3.8143897 & $\operatorname{chrX}$ & MIMAT0004775 \\
\hline & hsa-miR-5090 & -5.9943867 & -2.5836122 & $\operatorname{chr} 7$ & MIMAT0021082 \\
\hline & hsa-miR-5096 & -7.0916066 & -2.8261125 & $\operatorname{chr} 4$ & MIMAT0020603 \\
\hline & hsa-miR-513b & -3.0015473 & -1.5857065 & $\operatorname{chrX}$ & MIMAT0005788 \\
\hline & hsa-miR-516a-3p & -6.1154137 & -2.6124501 & chr19 & MIMAT0006778 \\
\hline & hsa-miR-5190 & -3.2782757 & -1.7129372 & chr18 & MIMAT0021121 \\
\hline & hsa-miR-5195-5p & -31.958092 & -4.9981093 & chr14 & MIMAT0021126 \\
\hline & hsa-miR-525-3p & -5.2564344 & -2.3940845 & chr19 & MIMAT0002839 \\
\hline & hsa-miR-532-3p & -31.67539 & -4.9852905 & $\operatorname{chrX}$ & MIMAT0004780 \\
\hline & hsa-miR-539-5p & -7.4776726 & -2.9025893 & $\operatorname{chr} 14$ & MIMAT0003163 \\
\hline & hsa-miR-543 & -31.493706 & -4.9769917 & chr14 & MIMAT0004954 \\
\hline & hsa-miR-574-3p & -2.0015302 & -1.0011034 & chr4 & MIMAT0003239 \\
\hline & hsa-miR-602 & -5.946781 & -2.572109 & chr9 & MIMAT0003270 \\
\hline & hsa-miR-6075 & -33.264282 & -5.055902 & chr5 & MIMAT0023700 \\
\hline & hsa-miR-622 & -6.371007 & -2.6715214 & $\operatorname{chr} 13$ & MIMAT0003291 \\
\hline & hsa-miR-6511b-5p & -7.128962 & -2.833692 & $\operatorname{chr16}$ & MIMAT0025847 \\
\hline & hsa-miR-6512-5p & -14.816795 & -3.8891616 & $\operatorname{chr} 2$ & MIMAT0025480 \\
\hline & hsa-miR-758-3p & -5.32387 & -2.4124753 & chr14 & MIMAT0003879 \\
\hline & hsa-miR-765 & -6.9221396 & -2.791218 & $\operatorname{chr} 1$ & MIMAT0003945 \\
\hline & hsa-miR-766-3p & -2.33501 & -1.2234287 & $\operatorname{chr} \mathrm{X}$ & MIMAT0003888 \\
\hline & hsa-miR-877-3p & -13.717739 & -3.7779708 & chr6 & MIMAT0004950 \\
\hline
\end{tabular}

to the KEGG database (20-22). The Fisher's exact test and chi-square test were used to select the significant pathway, and the threshold of significance was defined by P-value $(<0.05)$.

miRNA-pathway network analysis. A miRNA-pathway network was built according to the relationship among miRNAs and pathways as previously described (23).

Quantitative reverse transcriptase-polymerase chain reaction ( $q R T-P C R)$. qRT-PCR was performed to measure the levels of miRNAs. A total of $0.5 \mu \mathrm{g}$ of total RNA was reverse-transcribed using M-MLV reverse transcriptase (Thermo Fisher, Rockford, IL, USA) with a special stem-loop primer (Genepharma; Shanghai, China) for miRNAs. Real-time PCR was performed on ABI PRISM 7500 Real-time PCR system (Applied Biosystems; Foster City, CA, USA) using SYBR Green PCR kit (Thermo Fisher) according to manufacturer's instruction. All samples were analyzed in triplicate. The primer sequences were listed in Table I. The relative expression level was determined by the $2^{-\Delta \Delta \mathrm{Ct}}$ method and normalized to U6 expression. Statistical analysis was performed with ANOVA for multiple comparisons. P-value $<0.05$ were considered statistically significant.

\section{Results}

Identify differentially expressed miRNAs (DEMs). Placenta tissues around the individual insertion region for each umbilical cord were collected for RNA extraction and further analysis. Placenta tissues from two cases complicated with sIUGR [larger twin (L1 and L2), smaller twin (S1 and S2)] and one cases with normal MC [larger twin (N1) and smaller twin (n1)] were used for miRNA profile analysis by Affymetrix 
Table VI. DEMs (L1 vs. S1 and L2 vs. S2).

\begin{tabular}{|c|c|c|c|c|c|}
\hline Regulation & Systematic_name & $\begin{array}{c}\text { FC } \\
\text { (L1 vs. S1) }\end{array}$ & $\begin{array}{c}\text { FC } \\
(\mathrm{L} 2 \text { vs. S2) }\end{array}$ & Chromosome & $\begin{array}{c}\text { Mirbase } \\
\text { accession no. }\end{array}$ \\
\hline \multirow[t]{33}{*}{ Up-regulated } & hsa-miR-1 & 6.5372915 & 6.2799373 & $\operatorname{chr} 18$ & MIMAT0000416 \\
\hline & hsa-miR-101-3p & 7.129267 & 6.4381766 & $\operatorname{chrl}$ & MIMAT0000099 \\
\hline & hsa-miR-133b & 38.066643 & 37.049896 & chr6 & MIMAT0000770 \\
\hline & hsa-miR-154-3p & 6.04454 & 80.835304 & chr14 & MIMAT0000453 \\
\hline & hsa-miR-193a-3p & 31.360935 & 30.860113 & $\operatorname{chr17}$ & MIMAT0000459 \\
\hline & hsa-miR-218-5p & 6.466407 & 6.376806 & chr4 & MIMAT0000275 \\
\hline & hsa-miR-299-3p & 28.977842 & 30.239016 & chr14 & MIMAT0000687 \\
\hline & hsa-miR-3127-5p & 7.3825746 & 6.460783 & $\operatorname{chr} 2$ & MIMAT0014990 \\
\hline & hsa-miR-33b-3p & 6.059397 & 5.4492188 & $\operatorname{chr17}$ & MIMAT0004811 \\
\hline & hsa-miR-3620-5p & 88.913635 & 119.47107 & $\operatorname{chrl}$ & MIMAT0022967 \\
\hline & hsa-miR-3622b-5p & 6.9455557 & 6.797605 & chr8 & MIMAT0018005 \\
\hline & hsa-miR-370 & 6.1665797 & 6.585063 & chr14 & MIMAT0000722 \\
\hline & hsa-miR-411-5p & 7.6058702 & 6.842667 & chr14 & MIMAT0003329 \\
\hline & hsa-miR-4476 & 41.244473 & 6.292596 & $\operatorname{chr} 9$ & MIMAT0019003 \\
\hline & hsa-miR-4535 & 80.93671 & 84.8799 & $\operatorname{chr} 22$ & MIMAT0019075 \\
\hline & hsa-miR-4539 & 91.96671 & 156.92206 & chr14 & MIMAT0019082 \\
\hline & hsa-miR-4632-5p & 2.642979 & 6.771799 & chr1 & MIMAT0022977 \\
\hline & hsa-miR-4698 & 110.09238 & 122.85178 & $\operatorname{chr} 12$ & MIMAT0019793 \\
\hline & hsa-miR-4740-5p & 7.15094 & 140.27261 & $\operatorname{chr17}$ & MIMAT0019869 \\
\hline & hsa-miR-4743-5p & 41.097565 & 6.4767523 & $\operatorname{chr} 18$ & MIMAT0019874 \\
\hline & hsa-miR-4749-3p & 7.212792 & 6.195103 & chr19 & MIMAT0019886 \\
\hline & hsa-miR-487a & 32.68032 & 34.672806 & chr14 & MIMAT0002178 \\
\hline & hsa-miR-5003-3p & 74.188484 & 99.04469 & $\operatorname{chr} 5$ & MIMAT0021026 \\
\hline & hsa-miR-502-3p & 30.460754 & 6.4271126 & $\operatorname{chrX}$ & MIMAT0004775 \\
\hline & hsa-miR-5096 & 5.691079 & 28.428453 & chr4 & MIMAT0020603 \\
\hline & hsa-miR-513b & 2.4948008 & 5.130571 & $\operatorname{chrX}$ & MIMAT0005788 \\
\hline & hsa-miR-5189 & 7.34395 & 28.272223 & chr16 & MIMAT0021120 \\
\hline & hsa-miR-532-3p & 30.798903 & 6.8659596 & $\operatorname{chrX}$ & MIMAT0004780 \\
\hline & hsa-miR-539-5p & 7.7136526 & 28.060043 & chr14 & MIMAT0003163 \\
\hline & hsa-miR-543 & 37.697845 & 36.802776 & chr14 & MIMAT0004954 \\
\hline & hsa-miR-5581-5p & 2.7262392 & 69.77128 & chr1 & MIMAT0022275 \\
\hline & hsa-miR-6075 & 7.67851 & 35.023468 & $\operatorname{chr} 5$ & MIMAT0023700 \\
\hline & hsa-miR-887 & 33.127758 & 6.620651 & $\operatorname{chr} 5$ & MIMAT0004951 \\
\hline \multirow{12}{*}{$\begin{array}{l}\text { Down- } \\
\text { regulated }\end{array}$} & hsa-miR-335-3p & -4.238018 & -13.99673 & $\operatorname{chr} 7$ & MIMAT0004703 \\
\hline & hsa-miR-338-5p & -3.542421 & -11.96858 & $\operatorname{chr17}$ & MIMAT0004701 \\
\hline & hsa-miR-3653 & -3.068764 & -7.872367 & $\operatorname{chr} 22$ & MIMAT0018073 \\
\hline & hsa-miR-3679-3p & -4.817414 & -3.019187 & chr2 & MIMAT0018105 \\
\hline & hsa-miR-373-3p & -4.537483 & -3.249207 & chr19 & MIMAT0000726 \\
\hline & hsa-miR-4287 & -12.79339 & -2.985924 & chr8 & MIMAT0016917 \\
\hline & hsa-miR-4324 & -2.401632 & -3.439694 & chr19 & MIMAT0016876 \\
\hline & hsa-miR-4472 & -2.590619 & -3.141099 & $\operatorname{chr} 12$ & MIMAT0018999 \\
\hline & hsa-miR-590-5p & -3.056939 & -2.542061 & $\operatorname{chr} 7$ & MIMAT0003258 \\
\hline & hsa-miR-623 & -2.361158 & -3.541924 & $\operatorname{chr} 13$ & MIMAT0003292 \\
\hline & hsa-miR-664b-3p & -2.92985 & -3.077336 & $\operatorname{chrX}$ & MIMAT0022272 \\
\hline & hsa-miR-766-3p & -2.377063 & -7.31454 & $\operatorname{chrX}$ & MIMAT0003888 \\
\hline
\end{tabular}

microRNA 4.0 Array system. The expression of 2,578 miRNAs were examined. miRNAs with Fold Change $>=2.0$, and P-value $<0.05$ (g Student $\mathrm{t}$ test) were defines as DEMs.
Here, we identified a total of 130 (84 up-regulations and 46 down-regulations; Tables II and III) and 148 (107 up-regulations and 41 down-regulations; Tables II and 
Table VII. Target genes of potential target genes of 14 DEMs.

Regulation Systematic name

Up-regulated hsa-miR-1

hsa-miR-3622b-5p

hsa-miR-4535

hsa-miR-370-3p
Target genes

ABCA1, ABHD2, ABI2, ABL2, ACER2, ADAM12, ADAR, AKAP11, AMOT, AMOTL2, ANKIB1, ANKRD29, ANKRD34B, ANO1, ANP32B, ANXA4, AP3D1, API5, ARF3, ARHGEF18, ARID2, ASH2L, ASPH, BCL11A, BDNF, BET1, BLCAP, BMPR1B, BOLL, BSCL2, BSN, BZRAP1, C1RL, CAGE1, CALN1, CAPRIN1, CASK, CDC42, CEBPZ, CHM, CLCN3, CLTC, CNN3, COIL, COL4A3, CPEB1, CREB5, CREM, DDX5, DHX15, DICER1, DLG4, DNAJC5, E2F5, EHMT2, EIF1AX, EIF4E, EML3, EPB41L4B, ETS1, FAM107B, FAM126A, FAM134A, FAM155A, FAM168B, FAM46C, FAM63B, FAM91A1, FBXL14, FBXL20, FBXO22, FNDC3A, FOXP1, FRS2, FZD4, G6PD, GABBR2, GAS2L1, GCH1, GDF6, GJA1, GLCCI1, GLIS2, GMFB, GNPTAB, HACE1, HIAT1, HIGD1A, HMBOX1, HMGN1, HNRNPK, HNRNPU, HOOK1, HOXB4, HP1BP3, HS3ST3B1, HSP90B1, HSPD1, JARID2, KCNJ2, KCTD10, KDM5C, KDSR, KIAA1462, KTN1, LARP4, LASP1, LIN7C, LPPR4, LRCH1, LRRC8A, MAGI2, MAP3K1, MATR3, MEIS1, MEOX2, MET, MGAT4A, MIER1, MMD2, MMP8, MON2, MXD1, NAB1, NAMPT, NBEA, NCOA3, NDRG3, NET1, NFAT5, NR3C1, NR4A3, NRP1, NUP50, NXT2, OSBPL7, OSBPL8, OTX2, PABPC4L, PAX6, PAX7, PDE7A, PDGFA, PDIK1L, PFKFB2, PHAX, PHIP, PHLDA1, PKD2, PLEKHO2, POGK, PPIB, PREX1, PRIC285, PRKRIR, PTPLAD1, PTPN2, PTPRK, RAB43, RARB, RNF138, RNF141, RNF165, RNF213, RSBN1L, RUNX1, SEC22C, SEC23B, SEC63, SELT, SFRP1, SH3PXD2B, SH3TC2, SLC10A7, SLC16A6, SLC25A22, SLC25A30, SLC25A36, SLC29A3, SLC35B4, SLC35F1, SLC37A3, SLC39A9, SLC7A11, SLC8A2, SMAD4, SMAP1, SNED1, SNX13, SNX2, SOX9, SPRED1, SS18, STC2, STX12, SULF1, TAGLN2, TMED5, TMEM135, TMEM178, TMSB4X, TNKS2, TNPO1, TNRC6B, TNS3, TPPP, TRAPPC3, TRHDE, TRIM2, TTC3, TTC7B, UBE2H, UBE4A, UBR5, UTRN, VAMP2, WIPF2, WNK3, WSCD2, YPEL2, ZBTB4, ZBTB41, ZC3HAV1, ZFP91, ZNF148, ZNF236, ZNF652, ZZZ3

ANKRD52, ATRNL1, BEND4, CADM4, CBX5, CCDC34, CCDC97, CNKSR2, COL5A3, CPNE5, DCX, DVL3, EDEM3, EXTL3, FAM126B, FAM20B, FBXL20, FKBP5, FOXP3, GRIK2, HUWE1, KCTD20, KIAA0317, KIAA1239, KLF12, LARP1, LCORL, LOXL4, LPPR2, MAP3K3, MBOAT2, MIB1, MUM1L1, MYO1D, NDRG3, NTRK2, NUCKS1, NUP98, PAX6, PDE7B, PHF20L1, PHF21A, PTP4A1, PVRL1, PXT1, QKI, RIMKLA, SH3TC2, SLC1A2, SNTB2, SP2, SRGAP3, SSH2, STAG2, TBC1D14, TCF20, TRIM46, TRIM66, TSGA10, TSPAN11, VPS53, ZBTB7B

APBA1, CHD6, CLDN19, DNAJB12, EEF1A2, FKBP4, MAT2A, MYH7B, NDST1, PARVA, PTCD1, RIBC1, SCN2B, SPIN3, SPOPL, TUB ABCG4, ABR, ACCN4, ACOX1, ACTR1A, ACVR2B, ADCY5, AFF1, ANGEL1, ANKH, ANKRD52, ARCN1, ARF3, ASB10, ATP11A, ATP1A2, ATXN7L3, BAG4, BMF, BSN, C1QTNF6, CCDC64, CCL21, CDC42EP4, CFL1, CFLAR, CHD2, CHRNA7, CIT, CNGB1, CRLF1, CYB5B, CYP2U1, DES, DGCR14, DHX35, DMRTB1, DNAJB1, DNAJC11, DND1, EML1, ENAH, ENOX2, FAM102A, FAM123B, FAM164C, FAM168B, FBLN5, FBXO46, FGF11, FGF7, FOSL2, FOXO1, GADD45B, GSG1L, HDAC4, HEMK1, HHIPL1, HIF1AN, HNRNPUL2, HPS5, HSPA12A, HTR4, IKZF4, INO80, IPPK, IVD, JMY, KCNJ11, KIAA2018, KIF1B, KLC2, KLF12, KLHL18, KRT80, LPHN2, MAP2K7, MOCS1, MRPS25, MTL5, NAPG, NCDN, NCOA5, NEK9, NF1, NFASC, NLGN2, NTRK2, ODF2, OPA3, ORAI2, PACS1, PAPL, PCDH10, PCDH11X, PCDH19, PCLO, PDE7A, PHF19, PLEKHA6, PLEKHM1, POLR2F, POMT2, PPARGC1B, PRDM10, PRLR, PRRX1, PTCD1, PXMP4, RAB11A, RAP1GDS1, RAPGEF1, RAPGEFL1, RBBP4, SAP30BP, SEMA6A, SH3BP2, SHE, SLC10A7, SLC46A1, SLC4A4, SMURF1, SOX12, SPRYD3, ST3GAL3, ST6GAL1, STK35, SYNGR1, SYNPO2, TGFBR2, TM9SF4, TMCO7, TMEM127, TMEM154, TMEM184A, TMEM40, TNRC6C, TP53I11, TRIM33, TRIOBP, TRIT1, UBE2R2, UBTF, USP37, USP47, USP5, VANGL1, VSTM2L, WDTC1, WNT10B, ZBTB39, ZBTB42, ZC3H7B, ZC3HAV1, ZCCHC17, 
Table VII. Continued.

$\begin{array}{lll}\text { Regulation } \quad \text { Systematic name } & \text { Target genes }\end{array}$

hsa-miR-5189-5p AAK1, ACVR1B, ACVR2A, ADAT2, ADCY2, ADRBK1, AGBL4, AHI1, AKT2, ANKS6, ARHGAP19, ARID4A, ARRDC3, ASTN1, ASXL1, ATG4B, ATOH8, ATP2B2, ATP6V0D1, ATP8A1, BAIAP2L2, BAK1, BMP8B, BMPR2, BRAP, BRD4, BTRC, CA5B, CACNA1I, CACNB3, CALM3, CBFA2T2, CCBE1, CCDC69, CCDC76, CD300LG, CDH24, CHL1, CNP, CPLX2, CSMD2, CSRNP1, CYP26B1, DACT3, DCBLD2, DCHS1, DENND1A, DIS3L2, DNAJC5G, DTX1, DUOX1, EEFSEC, ELFN2, FAM105B, FAM120C, FAM155B, FAM53C, FBXL19, FBXL20, FBXO33, FBXO41, FGD3, FGF14, FOXN3, FOXP1, GGT5, GLG1, GLUL, GNA12, GRIN1, GRIP2, GRK6, HEG1, HM13, HOXA13, IGFN1, KATNB1, KCNK2, KCTD15, KIF21B, KPNA6, LCNL1, LHFPL4, LHX6, LHX8, LMAN2, LRRTM3, LYPLA2, LZTR1, M6PR, MAP3K3, MAPK1IP1L, MARCKSL1, MCTS1, MDGA1, MECP2, MLLT6, MMP19, MPP2, MYO1D, NAGS, NAT8L, NAV1, NDRG4, NFYA, NIPSNAP1, NOL3, NPTX1, NRP1, NUAK1, NUP43, OBFC1, OFD1, OSBPL7, PBX1, PCDH11X, PHF15, PHF21A, PIGA, PLEKHM3, PODXL, POLDIP2, POLR2F, PPIL6, PPME1, PRMT2, PROSC, PTOV1, RAB11FIP3, RAB11FIP4, RAB11FIP5, RAB22A, RC3H1, REM1, RFX1, RHBDL3, RIMS3, RNF157, RNMTL1, RUNX3, SEC14L1, SEMA3G, SENP5, SETBP1, SH3PXD2B, SHANK2, SHANK3, SLC17A5, SLC23A2, SLC26A9, SLC30A6, SLC38A3, SLC6A4, SMARCD1, SNCA, SNX27, SRF, SRRM1, SS18L1, ST3GAL3, ST7L, STAC3, STK4, STX1B, SUV39H1, SV2A, SYNPO, SYT9, TBC1D13, TCF7L1, TFAP2A, THRB, TLN2, TMEM79, TRIM10, TRIM16, TRIM44, TRIM9, TSG101, TSPAN18, TSR2, TTBK1, USH1G, USP54, VAMP2, VCPIP1, VPS39, WBSCR17, WDR37, WDTC1, WTAP, XYLT1, YEATS2, ZBTB7B, ZER1, ZHX3, ZNF76, ZNRF1

hsa-miR-4743-5p hsa-miR-5581-5p

Down-regulated hsa-miR-373-3p
AKT1S1, ARL3, GRIN1, HIC1, NCDN, OLFML2A, SCRT2, ZDHHC8 APLNR, ATP6V1A, ATP8A1, BRD4, BTG4, CABP7, CADM1, CALN1, CAPN1, CCDC62, CCL22, CDON, CHRNB2, CLDN2, CLIC4, CPEB2, CR2, CSNK1D, CTNND2, DCLK2, DRP2, FAF2, FAM13A, FGA, FNDC5, FOXP1, GGA1, GMEB1, GRIN1, HIF3A, HNRNPA3, IFFO2, IL4R, IPO7, ITPKB, KCNK3, KPNA6, LAMC1, LHX6, LIPH, MMP19, MTHFR, MYO5A, NACC1, NCOA3, NEDD4L, NTN1, NWD1, PARP16, PHF8, PHOSPHO1, PLCB3, PNKD, RALGPS1, RECQL5, RIMKLA, RMND5A, RNF169, SH3PXD2A, SHROOM4, SLC26A9, SYNGR3, SYT11, TBRG1, TGFBR1, THSD7A, TP53111, TPM3, TRIM47, TUB, UBAP2L, UBXN7, UNC119, VEZF1, ZKSCAN2, ZNF304, ZNF576, ZNF608, ZNF629, ZXDC

A2LD1, AAK1, ABCA1, ABHD3, ABI2, ABL2, ACBD5, ACVR1C, ADAM9, ADAMTS18, AFF2, AGAP2, AHNAK, AKAP5, AKTIP, ANKRD13C, ANKRD50, ANKRD52, ANO6, AP1M1, APBB2, ARHGAP30, ARHGEF10, ARHGEF18, ARHGEF3, ARHGEF7, ARID4A, ARID4B, ARL4A, ASAP1, ASB1, ASF1B, ASH1L, ATP2B2, ATXN1, BAHD1, BAMBI, BCAT1, BCL11A, BCL11B, BCL2L11, BCL6B, BMPR2, BNC2, BNIP3L, BRMS1L, BRWD1, BSCL2, BTBD7, BVES, CAMSAP1, CAMTA1, CASC4, CC2D1A, CCDC88A, CCND1, CCND2, CD44, CDC25A, CDC40, CDCA7, CFL2, CHD9, CLIP4, CNN1, CNOT6, CORIN, CREB1, CRK, CROT, CXCL12, CXCL14, CYB561D1, CYBB, CYBRD1, CYP26B1, DDHD1, DENND5B, DERL2, DGKE, DIRC2, DLGAP2, DMTF1, DNAJA2, DNAJC27, DPP3, DPP8, DPYSL5, DYNLT3, EDNRB, EGLN1, EIF4B, ELAVL2, ENDOD1, EPHA2, EPHA5, EPHA7, ERO1LB, EZH1, FAM102B, FAM117A, FAM18B2, FAM40B, FAM46C, FBXL4, FBXO10, FBXO41, FGD4, FGD5, FLT1, FMNL3, FOXK2, FOXO3, FRMD4A, FRMD4B, FYCO1, FZD6, GAB2, GALNT10, GALNT3, GATAD2B, GATC, GDA, GLIS3, GLS, GNB5, GNG12, GNPDA2, GOLGA1, GPR12, GPR137C, GPR180, GUCY1A3, HAUS8, HDAC4, HEG1, HIP1, HIPK3, HK1, HLF, HMGXB3, HN1, HNRNPUL2, HOOK3, HP1BP3, IGDCC3, IKZF2, IL28RA, IL8, INO80D, IPO7, IQSEC1, IRAK2, IRAK4, IRF2, IRF9, ISM1, ITGB8, 
Table VII. Continued.

JUB, KDM2A, KIAA0226, KIAA0240, KIAA0513, KIAA1522, KIAA1549, KIAA1737, KIF3B, KLF12, KLF13, KLF3, KLHL28, KREMEN1, KSR2, LEF1, LEFTY1, LEFTY2, LHX6, LHX8, LIF, LMO3, LRIT1, LUC7L2, LYPD6, LYRM2, LYSMD3, LYST, MAML1, MAP1B, MAP3K1, MAP3K14, MAP3K2, MBD2, MBNL2, MBNL3, MCCD1, MCL1, MDM4, MECP2, MED13L, MFAP3L, MIB1, MICAL3, MKNK2, MKRN1, MLL, MLL3, MLLT6, MNT, MRPS25, MSL1, MTCH2, MTF1, MTMR3, MTUS1, MYO1D, NAPEPLD, NCOA3, NCOA7, NECAP1, NEK9, NFATC3, NFIB, NFYA, NHLRC2, NHLRC3, NNAT, NPAS3, NR2C1, NR2C2, OCRL, ODF2, OPCML, ORMDL3, OSBPL5, OSTM1, OTUD7B, PAFAH2, PAG1, PAK2, PAM, PAN3, PARP8, PBX3, PCDH7, PCGF5, PDCD4, PDLIM5, PFN2, PGBD5, PHACTR4, PHC3, PHF6, PHKA1, PHYHIPL, PIP4K2A, PKD2, PKN2, PLAG1, PLCL1, POFUT1, POLK, POU6F1, PPARA, PPARGC1B, PPP1R10, PPP1R9A, PPP6C, PRDM16, PRDM8, PRKACB, PRMT6, PRRT2, PRRX1, PSD3, PSEN1, PTGDR, PTPDC1, RAB11A, RAB11FIP1，RAB11FIP5, RAB22A, RABEP1，RAD18，RAD23B, RALGDS, RAPGEF2, RAPGEF5, RAPGEFL1, RASSF2, RBL1, RBMS2, RDX, RELA, RELL1, RGL1, RGMA, RHOC, RIMKLA, RNF180, RNF216, RNF38, RNF6, RORA, RPS6KA2, RRAGD, RSBN1, RSBN1L, RSF1, RSRC2, RUNX2, RYR2, SAMD12, SAR1B, SASH1, SBF1, SCD5, SCN2A, SCN5A, SCRT2, SDC1, SETBP1, SETD7, SHCBP1, SIK1, SIPA1L3, SLC14A1, SLC16A12, SLC16A9, SLC35E1, SLC38A1, SLC39A6, SLC46A3, SLC6A9, SMARCC2, SNRK, SNTB2, SNX30, SNX5, SNX9, SOS1, SPRED1, SS18L1, SSX2IP, ST3GAL5, ST8SIA2, STX16, SUV420H2, SYAP1, SYDE1, SYNC, SYNPO2, TANC2, TAOK2, TAPT1, TARDBP, TBCEL, TCEB3, TET2, TET3, TGFBR2, TIAM1, TMCC1, TMEM100, TMTC2, TMUB2, TNRC18, TNRC6B, TNRC6C, TNS1, TOX, TRAPPC2, TRHDE, TRIM2, TRIM44, TRIM66, TRPS1, TRPV6, TSEN34, TSHZ3, TTC9, TTPAL, TUSC2, UBASH3B, UBE2B, UBE2J1, UBE2Q2, UBE2R2, UBE2W, UBN1, UBN2, UHRF1, UHRF1BP1, ULK1, UNK, UNKL, UPF3A, USP24, USP42, USP46, USP53, VSX1, WDR26, WDR37, WDR45, WEE1, WIPF2, YTHDF3, ZBTB11, ZBTB41, ZBTB43, ZBTB44, ZBTB47, ZBTB7A, ZCCHC24, ZDHHC8, ZDHHC9, ZFP91, ZFYVE26, ZKSCAN1, ZMYND11, ZNF148, ZNF2, ZNF236, ZNF25, ZNF292, ZNF362, ZNF385A, ZNF436, ZNF473, ZNF512B, ZNF518A, ZNF566, ZNF597, ZNF697, ZNF862, ZNFX1"

hsa-miR-4287 AKT2, AP3M2, APLN, ASTN1, ATG9A, BAHD1, BHLHE41, BSDC1, BTG2, CALB1, CAMK2A, CAMK2B, CCDC113, CECR6, COL17A1, CRTC2, DDX3X, DDX3Y, DNAJC21, EHF, EIF2S1, ENC1, EYA3, FAM117B, FAM76A, GCC1, GRAMD4, HELZ, HUNK, IGSF9B, KCNA6, KCNK10, KIAA1210, KLF12, KPNA6, KRT80, MDM1, MFAP3L, MID1, NARG2, NBN, NCAN, NFASC, OPCML, ORAI3, OSBP, PDE1B, PHF23, PI4K2A, PIK3C2B, PMEPA1, POLD3, RAB1B, RGL1, RIPK1, ROBO2, SGCZ, SGTB, SH3BP2, SH3RF2, TIGD3, TIMM17B, TOX2, UBN2, VBP1, ZNF48, ZNRF3, CREB1, CRK, CROT, CXCL12, CXCL14, CYB561D1, CYBB, CYBRD1, CYP26B1, DDHD1, DENND5B, DERL2, DGKE, DIRC2, DLGAP2, DMTF1, DNAJA2, DNAJC27, DPP3, DPP8, DPYSL5, DYNLT3, EDNRB, EGLN1, EIF4B, ELAVL2, ENDOD1, EPHA2, EPHA5, EPHA7, ERO1LB, EZH1, FAM102B, FAM117A, FAM18B2, FAM40B, FAM46C, FBXL4, FBXO10, FBXO41, FGD4, FGD5, FLT1, FMNL3, FOXK2, FOXO3, FRMD4A, FRMD4B, FYCO1, FZD6, GAB2, GALNT10, GALNT3, GATAD2B, GATC, GDA, GLIS3, GLS, GNB5, GNG12, GNPDA2, GOLGA1, GPR12, GPR137C, GPR180, GUCY1A3, HAUS8, HDAC4, HEG1, HIP1, HIPK3, HK1, HLF, HMGXB3, HN1, HNRNPUL2, HOOK3, HP1BP3, IGDCC3, IKZF2, IL28RA, IL8, INO80D, IPO7, IQSEC1, IRAK2, IRAK4, IRF2, IRF9, ISM1, ITGB8, JUB, KDM2A, KIAA0226, KIAA0240, KIAA0513, KIAA1522, KIAA1549, KIAA1737, KIF3B, KLF12, KLF13, KLF3, KLHL28, KREMEN1, KSR2, LEF1, LEFTY1, LEFTY2, LHX6, LHX8, LIF, LMO3, LRIT1, LUC7L2, LYPD6, LYRM2, LYSMD3, LYST, MAML1, 
Table VII. Continued.

\begin{tabular}{lll}
\hline Regulation & Systematic name & Target genes \\
\hline
\end{tabular}

MAP1B, MAP3K1, MAP3K14, MAP3K2, MBD2, MBNL2, MBNL3, MCCD1, MCL1, MDM4, MECP2, MED13L, MFAP3L, MIB1, MICAL3, MKNK2, MKRN1, MLL, MLL3, MLLT6, MNT, MRPS25, MSL1, MTCH2, MTF1, MTMR3, MTUS1, MYO1D, NAPEPLD, NCOA3, NCOA7, NECAP1, NEK9, NFATC3, NFIB, NFYA, NHLRC2, NHLRC3, NNAT, NPAS3, NR2C1, NR2C2, OCRL, ODF2, OPCML, ORMDL3, OSBPL5, OSTM1, OTUD7B, PAFAH2, PAG1, PAK2, PAM, PAN3, PARP8, PBX3, PCDH7, PCGF5, PDCD4, PDLIM5, PFN2, PGBD5, PHACTR4, PHC3, PHF6, PHKA1, PHYHIPL, PIP4K2A, PKD2, PKN2, PlAG1, PLCL1, POFUT1, POLK, POU6F1, PPARA, PPARGC1B, PPP1R10, PPP1R9A, PPP6C, PRDM16, PRDM8, PRKACB, PRMT6, PRRT2, PRRX1, PSD3, PSEN1, PTGDR, PTPDC1, RAB11A, RAB11FIP1, RAB11FIP5, RAB22A, RABEP1, RAD18, RAD23B, RALGDS, RAPGEF2, RAPGEF5, RAPGEFL1, RASSF2, RBL1, RBMS2, RDX, RELA, RELL1, RGL1, RGMA, RHOC, RIMKLA, RNF180, RNF216, RNF38, RNF6, RORA, RPS6KA2, RRAGD, RSBN1, RSBN1L, RSF1, RSRC2, RUNX2, RYR2, SAMD12, SAR1B, SASH1, SBF1, SCD5, SCN2A, SCN5A, SCRT2, SDC1, SETBP1, SETD7, SHCBP1, SIK1, SIPA1L3, SLC14A1, SLC16A12, SLC16A9, SLC35E1, SLC38A1, SLC39A6, SLC46A3, SLC6A9, SMARCC2, SNRK, SNTB2, SNX30, SNX5, SNX9, SOS1, SPRED1, SS18L1, SSX2IP, ST3GAL5, ST8SIA2, STX16, SUV420H2, SYAP1, SYDE1, SYNC, SYNPO2, TANC2, TAOK2, TAPT1, TARDBP, TBCEL, TCEB3, TET2, TET3, TGFBR2, TIAM1, TMCC1, TMEM100, TMTC2, TMUB2, TNRC18, TNRC6B, TNRC6C, TNS1, TOX, TRAPPC2, TRHDE, TRIM2, TRIM44, TRIM66, TRPS1, TRPV6, TSEN34, TSHZ3, TTC9, TTPAL, TUSC2, UBASH3B, UBE2B, UBE2J1, UBE2Q2, UBE2R2, UBE2W, UBN1, UBN2, UHRF1, UHRF1BP1, ULK1, UNK, UNKL, UPF3A, USP24, USP42, USP46, USP53, VSX1, WDR26, WDR37, WDR45, WEE1, WIPF2, YTHDF3, ZBTB11, ZBTB41, ZBTB43, ZBTB44, ZBTB47, ZBTB7A, ZCCHC24, ZDHHC8, ZDHHC9, ZFP91, ZFYVE26, ZKSCAN1, ZMYND11, ZNF148, ZNF2, ZNF236, ZNF25, ZNF292, ZNF362, ZNF385A, ZNF436, ZNF473, ZNF512B, ZNF518A, ZNF566, ZNF597, ZNF697, ZNF862, ZNFX1

hsa-miR-338-5p AAK1, ADAMTS17, ADARB2, AEBP2, AMMECR1, APPL1, ARFGAP3, ARID2, ARNT, ATAD1, ATF7, ATP2C1, ATRX, AUTS2, B4GALT6, BAZ1B, BCL11B, BCL2L11, BTG3, CADM2, CALM3, CAST, CCDC140, CCNT2, CD28, CD82, CD9, CDK5R1, CDYL2, CHST12, CLIC4, CLTC, CNR1, CNTN4, CPEB4, CPNE3, CREB3L1, CRIM1, CSNK1G1, CUL3, DGKG, DICER1, DLAT, DMXL2, DNAJC6, DNM3, DYRK4, EML1, EP300, EPAS1, EPHA7, ERRFI1, EXOC5, FAM126A, FAM129B, FAM135B, FAM177A1, FAM18B2, FMNL2, FNDC3B, FOXJ3, FUT9, GATAD2B, GREM2, GRIA4, GRM7, GTF3C2, GUCY1A3, HCN1, HDAC9, HIF1A, HIPK2, HSPA12A, IKZF1, IMPACT, INO80D, IREB2, JMJD1C, KAL1, KDM5B, KIAA1024, KIAA1467, KLF11, KLHL14, KLHL6, KLRAQ1, KRAS, LMO4, LRP1, MACF1, MBNL1, MBNL2, MCTS1, MEF2C, MIPOL1, MKL2, MLL4, MLLT4, MN1, MON2, MPPED2, NCK2, NCOA3, NDFIP1, NPAS4, NRP1, NUDT4, NUFIP2, OCIAD1, ONECUT2, PARD6B, PCDH17, PCDH20, PCGF5, PCNX, PELI1, PHC3, PHIP, PKN2, PLAGL2, PLEKHA5, PPARGC1A, PPM1B, PPP2R5A, PRDM10, PRLR, PTCHD1, PTGS1, R3HDM2, RAB14, RAB1A, RAB22A, RAB6B, RAP2C, RAPGEF5, RAPH1, RCOR1, RICTOR, RND3, RNF138, RORA, SAMD12, SBNO1, SEC16B, SEMA6A, SERTAD2, SIRT1, SKP1, SLC4A7, SLIT1, SLMAP, SNTB1, SOX6, SPAST, SPOP, SSX2IP, STAG2, SUB1, SYNCRIP, SYPL1, TAF4, TANC2, TARDBP, TBX18, TBX2, TCERG1L, TEAD1, TET2, TLK1, TRA2B, TRAF3, TRPM7, TSHZ3, UBE2N, UBR2, USP25, WASF1, WDFY3, WWC3, ZBTB44, ZFAND5, ZNF292

hsa-miR-623 AAK1, ACSM2A, ADARB2, AGPAT4, ALPL, AP3M2, APPL1, ATG9A, BAHD1, CACNA1C, CAMK2B, CCDC117, CCDC3, CELSR3, CLUAP1, CORO2A, CRTC2, CXCL12, DCLK1, DCLK3, DSEL, ECE1, EGFLAM, 
Table VII. Continued.

\begin{tabular}{lll}
\hline Regulation & Systematic name & Target genes
\end{tabular}

EIF1, ELAVL2, EZR, FAM126A, FAM134C, FOXN2, GATAD2B, GLIS3, HAS3, HGSNAT, HLCS, HM13, HMGA2, HOXC10, HOXC9, IGF2R, ILDR2, KIAA1199, KPNA1, MAPK1, MECP2, MEIS1, MFSD11, MON2, MTMR7, NIPBL, NMT1, NR3C2, NTRK2, NTRK3, OBFC2A, ODZ4, PCMT1, PDE4A, PI4KB, PLCD4, POLD3, PRIMA1, RBM24, RHOBTB3, RIMKLA, RIN3, RNF144A, RNF169, RPRD2, SECISBP2L, SH3PXD2A, SH3TC2, SIGLEC1, SKI, SLC12A2, SLC44A5, SNX13, SUPT16H, TAOK2, TET3, TNFRSF8, TNRC6B, TPM3, TRIM31, TRPS1, ZMIZ1

hsa-miR-3653 ACVR1C, ADCY2, AEBP2, AMIGO2, ATP1B4, ATRNL1, ATXN7, BMP3, BMPR2, BNC2, BRD3, BRPF3, BRWD3, BTG1, CCDC88A, CPEB4, DBT, DGCR2, DIXDC1, DUSP19, EFNB3, ESRRB, FAM107B, FASLG, GALNT2, GJC1, GPC2, GPC6, HCFC2, HIPK3, KIAA0947, KIAA2018, KLHL28, LPCAT2, LRRTM2, MED12L, MKLN1, MYSM1, NCOA1, NIP7, ODZ3, PCDH11X, PDE11A, PHLDA1, PI15, PRPF4B, R3HDM1, RBBP4, SEC62, SERBP1, SORT1, SPATA5, SV2B, TMEM215, TMEM50B, TRIM67, TRPM8, VPS33A, YAF2, ZADH2, ZDHHC21, ZFAND5, ZFY, ZNF280C, ZNF507, ZYG11B

hsa-miR-590-5p ARHGAP24, ARHGEF12, ARMCX1, BAHD1, BMP3, BMPR2, CADM1, CCL22, CEP68, CNOT6, CREB5, DAG1, DSC2, EIF2C4, EIF4EBP2, ELF2, ENAH, EPHA4, FAM13A, FAM3C, FASLG, FBXO28, FGD4, FGF1, FRS2, GABRB2, GATAD2B, GLCCI1, GPR64, ITGB8, JHDM1D, JPH1, KCNT2, KLF12, KLHDC5, LCORL, LRRC57, MATN2, MBNL1, MICALL1, MTMR12, NELL2, NFAT5, NFIB, OSR1, PAG1, PAIP2B, PAN3, PBRM1, PCBP2, PDZD2, PER2, PGRMC2, PIK3R1, PLAG1, PLEKHA1, PPP1R3B, PTPN9, RAB22A, RASGRP1, RAVER2, RBPJ, RECK, RFFL, RP2, RPRD1A, SATB1, SECISBP2L, SESTD1, SETD1B, SKI, SLC7A6, SNTB2, SNX29, SPRY2, ST3GAL6, STAG2, TAGAP, TBX2, TET1, TGFB2, TGFBR2, TNRC6B, UBE2D3, UBN2, UBR3, YOD1, ZCCHC3, ZNF704

hsa-miR-664b-3p AASS, ABCE1, ABI2, ACVR2B, ADD3, AKNA, APAF1, APC, ARPM1, ASB13, ATG7, ATP2C1, BACH1, BACH2, BCAS1, BDNF, BHLHB9, BNC2, CA5B, CACHD1, CCNC, CENPL, COPA, CREG2, DCP2, DENND4C, DIP2B, DPY19L1, EDAR, ERBB4, ETNK1, FAM114A1, FAM8A1, FBXW2, FNDC3A, FRMD4A, FZD5, GPRASP2, HIPK2, HMGA2, INTS6, JPH1, KCTD21, KLF12, KLHDC10, LDB3, LMTK3, MED1, MSR1, MTCH2, MTR, MYCBP, MYO1D, N4BP2, NDFIP1, NETO1, NFIB, NIPAL3, NOTCH2, NSL1, NUFIP2, PAPD5, PARVA, PDE4D, PDYN, PELI2, PGM3, PHIP, PI15, PKP1, PRDM10, PRDM15, PRKAA1, PRKAA2, PRLR, QKI, RANBP9, RAPGEF6, RIBC1, RS1, RSU1, SALL4, SAMD12, SH2D4B, SMAD3, SNX30, STT3A, TAF4, TGOLN2, TMEM215, TMEM26, TRAM2, TXLNA, UTP23, VAMP1, VAMP4, WDFY1, WWC2, YKT6, ZFHX3, ZFP28, ZFX, ZNF24

IV) significantly DEMs in L1 and L2, respectively, when compared with S1 and S2. A total of 133 significantly DEMs with 50 up-regulations and 83 down-regulations; Tables II and $\mathrm{V}$ ) were identified in N1, when compared with $\mathrm{n} 1$. As shown in Fig. 1 and Table VI, 45 DEMs (33 up-regulators and 12 down-regulators) identified from L1 vs. S1 were included in the list of DEMs identified from L2 and S2 comparison. More importantly, 7 up-regulated miRNAs and 7 down-regulated miRNAs identified from the territory of sIUGR larger twins vs. sIUGR smaller twins (L1 vs. S1 and L2 vs. S2) were not included in the list of DEMs identified from N1 and $\mathrm{n} 1$ (Figs. 1B and 2). These 14 DEMs may be associated with the pathology of sIUGR, and then subjected to target gene analysis, pathway analysis and miRNA-pathway analysis.

Pathway analysis. The potential target genes of the above 14 DEMs were then searched by using bioinformatic algorithms such as MiRanda and TargetScan. There are 712 and 929 target genes for up-regulated and down-regulated DEMs, respectively, and listed in Table VII.

To find out the significant pathway associated with the target genes, pathway analysis was performed according to the KEGG database. The results showed that 49 and 101 significant pathways were associated with the up-regulated and down-regulated DEMs, respectively $(\mathrm{P}<0.05$; Table VIII 
Table VIII. Pathway analysis based on miRNA-targeted genes.

\begin{tabular}{|c|c|c|c|c|c|c|}
\hline Regulation & Name & $\begin{array}{l}\text { Diffgene } \\
\text { count }\end{array}$ & $\begin{array}{l}\text { Gene } \\
\text { count }\end{array}$ & Enrichment & P-value & FDR \\
\hline \multirow[t]{46}{*}{ Up-regulated } & Pathways in cancer & 23 & 327 & 4.3892 & $1.822 \mathrm{E}-08$ & $2.670 \mathrm{E}-06$ \\
\hline & TGF-beta signaling pathway & 12 & 81 & 9.2448 & 2.697E-08 & $2.670 \mathrm{E}-06$ \\
\hline & MAPK signaling pathway & 20 & 260 & 4.8002 & 4.116E-08 & $2.716 \mathrm{E}-06$ \\
\hline & Hippo signaling pathway & 15 & 156 & 6.0002 & $1.526 \mathrm{E}-07$ & 7.555E-06 \\
\hline & Endocytosis & 16 & 204 & 4.8943 & $9.288 \mathrm{E}-07$ & $3.678 \mathrm{E}-05$ \\
\hline & HTLV-I infection & 18 & 268 & 4.1912 & $1.689 \mathrm{E}-06$ & $5.371 \mathrm{E}-05$ \\
\hline & Glutamatergic synapse & 12 & 118 & 6.3460 & 1.899E-06 & $5.371 \mathrm{E}-05$ \\
\hline & Estrogen signaling pathway & 10 & 100 & 6.2403 & $1.987 \mathrm{E}-05$ & 4.917E-04 \\
\hline & $\begin{array}{l}\text { Protein processing in } \\
\text { endoplasmic reticulum }\end{array}$ & 12 & 167 & 4.4840 & 7.169E-05 & $1.577 \mathrm{E}-03$ \\
\hline & Neurotrophin signaling pathway & 10 & 120 & 5.2002 & $1.001 \mathrm{E}-04$ & $1.981 \mathrm{E}-03$ \\
\hline & $\begin{array}{l}\text { Transcriptional misregulation } \\
\text { in cancer }\end{array}$ & 12 & 180 & 4.1602 & $1.505 \mathrm{E}-04$ & 2.709E-03 \\
\hline & Insulin secretion & 8 & 87 & 5.7382 & $3.211 \mathrm{E}-04$ & $5.297 \mathrm{E}-03$ \\
\hline & Wnt signaling pathway & 10 & 143 & 4.3638 & 4.435E-04 & $6.350 \mathrm{E}-03$ \\
\hline & GnRH signaling pathway & 8 & 92 & 5.4263 & 4.769E-04 & $6.350 \mathrm{E}-03$ \\
\hline & $\begin{array}{l}\text { Cytokine-cytokine receptor } \\
\text { interaction }\end{array}$ & 14 & 267 & 3.2720 & $4.811 \mathrm{E}-04$ & $6.350 \mathrm{E}-03$ \\
\hline & Adherens junction & 7 & 73 & 5.9838 & $6.869 \mathrm{E}-04$ & $8.495 \mathrm{E}-03$ \\
\hline & Calcium signaling pathway & 11 & 183 & 3.7510 & 7.844E-04 & $8.495 \mathrm{E}-03$ \\
\hline & Gastric acid secretion & 7 & 75 & 5.8242 & 8.141E-04 & $8.495 \mathrm{E}-03$ \\
\hline & Regulation of actin cytoskeleton & 12 & 215 & 3.4829 & $8.152 \mathrm{E}-04$ & $8.495 \mathrm{E}-03$ \\
\hline & Melanogenesis & 8 & 101 & 4.9428 & $9.132 \mathrm{E}-04$ & $9.040 \mathrm{E}-03$ \\
\hline & Axon guidance & 9 & 131 & 4.2872 & $1.105 \mathrm{E}-03$ & $1.042 \mathrm{E}-02$ \\
\hline & RNA transport & 10 & 165 & 3.7820 & $1.418 \mathrm{E}-03$ & $1.276 \mathrm{E}-02$ \\
\hline & Ubiquitin mediated proteolysis & 9 & 138 & 4.0697 & $1.623 \mathrm{E}-03$ & $1.398 \mathrm{E}-02$ \\
\hline & Cholinergic synapse & 8 & 113 & 4.4179 & $1.956 \mathrm{E}-03$ & $1.614 \mathrm{E}-02$ \\
\hline & $\begin{array}{l}\text { Glycosaminoglycan biosynthesis- } \\
\text { heparan sulfate/heparin }\end{array}$ & 4 & 24 & 10.4004 & $2.153 \mathrm{E}-03$ & $1.705 \mathrm{E}-02$ \\
\hline & Synaptic vesicle cycle & 6 & 64 & 5.8502 & $2.259 \mathrm{E}-03$ & $1.721 \mathrm{E}-02$ \\
\hline & Salivary secretion & 7 & 90 & 4.8535 & 2.493E-03 & $1.829 \mathrm{E}-02$ \\
\hline & Morphine addiction & 7 & 93 & 4.6970 & $3.034 \mathrm{E}-03$ & $2.146 \mathrm{E}-02$ \\
\hline & Pancreatic secretion & 7 & 96 & 4.5502 & 3.664E-03 & $2.502 \mathrm{E}-02$ \\
\hline & Melanoma & 6 & 71 & 5.2735 & $3.928 \mathrm{E}-03$ & $2.592 \mathrm{E}-02$ \\
\hline & Chemokine signaling pathway & 10 & 192 & 3.2501 & $4.581 \mathrm{E}-03$ & $2.926 \mathrm{E}-02$ \\
\hline & Cocaine addiction & 5 & 50 & 6.2403 & 4.868E-03 & $3.012 \mathrm{E}-02$ \\
\hline & PI3K-Akt signaling pathway & 14 & 347 & 2.5177 & $6.421 \mathrm{E}-03$ & $3.853 \mathrm{E}-02$ \\
\hline & Focal adhesion & 10 & 206 & 3.0293 & $7.726 \mathrm{E}-03$ & 4.499E-02 \\
\hline & Gap junction & 6 & 89 & 4.2069 & $1.252 \mathrm{E}-02$ & $6.800 \mathrm{E}-02$ \\
\hline & Prostate cancer & 6 & 89 & 4.2069 & $1.252 \mathrm{E}-02$ & $6.800 \mathrm{E}-02$ \\
\hline & Colorectal cancer & 5 & 62 & 5.0325 & $1.271 \mathrm{E}-02$ & $6.800 \mathrm{E}-02$ \\
\hline & Lysosome & 7 & 122 & 3.5805 & $1.444 \mathrm{E}-02$ & 7.524E-02 \\
\hline & Proteoglycans in cancer & 10 & 227 & 2.7490 & $1.550 \mathrm{E}-02$ & $7.868 \mathrm{E}-02$ \\
\hline & Renal cell carcinoma & 5 & 66 & 4.7275 & $1.666 \mathrm{E}-02$ & $8.044 \mathrm{E}-02$ \\
\hline & Pancreatic cancer & 5 & 66 & 4.7275 & $1.666 \mathrm{E}-02$ & $8.044 \mathrm{E}-02$ \\
\hline & Circadian entrainment & 6 & 97 & 3.8600 & $1.913 \mathrm{E}-02$ & $9.018 \mathrm{E}-02$ \\
\hline & $\begin{array}{l}\text { Proximal tubule bicarbonate } \\
\text { reclamation }\end{array}$ & 3 & 23 & 8.1395 & $2.286 \mathrm{E}-02$ & $1.053 \mathrm{E}-01$ \\
\hline & Tight junction & 7 & 134 & 3.2598 & $2.404 \mathrm{E}-02$ & $1.082 \mathrm{E}-01$ \\
\hline & Chronic myeloid leukemia & 5 & 73 & 4.2741 & $2.556 \mathrm{E}-02$ & $1.125 \mathrm{E}-01$ \\
\hline & Endocrine and other factor-regulated & 4 & 49 & 5.0941 & $3.133 \mathrm{E}-02$ & $1.348 \mathrm{E}-01$ \\
\hline
\end{tabular}


Table VIII. Continued.

\begin{tabular}{|c|c|c|c|c|c|c|}
\hline Regulation & Name & $\begin{array}{l}\text { Diffgene } \\
\text { count }\end{array}$ & $\begin{array}{l}\text { Gene } \\
\text { count }\end{array}$ & Enrichment & P-value & FDR \\
\hline & calcium reabsorption & & & & & \\
\hline & Cell adhesion molecules (CAMs) & 7 & 146 & 2.9919 & 3.776E-02 & $1.591 \mathrm{E}-01$ \\
\hline & Basal cell carcinoma & 4 & 55 & 4.5384 & 4.679E-02 & $1.930 \mathrm{E}-01$ \\
\hline & Other types of O-glycan biosynthesis & 3 & 30 & 6.2403 & 4.825E-02 & $1.950 \mathrm{E}-01$ \\
\hline \multirow[t]{46}{*}{ Down-regulated } & Neurotrophin signaling pathway & 22 & 120 & 8.8478 & $2.651 \mathrm{E}-14$ & $5.567 \mathrm{E}-12$ \\
\hline & Proteoglycans in cancer & 26 & 227 & 5.5277 & 9.364E-12 & $9.832 \mathrm{E}-10$ \\
\hline & Axon guidance & 19 & 131 & 6.9996 & $1.365 \mathrm{E}-10$ & 8.205E-09 \\
\hline & Hepatitis B & 20 & 148 & 6.5217 & $1.563 \mathrm{E}-10$ & 8.205E-09 \\
\hline & MAPK signaling pathway & 26 & 260 & 4.8261 & $2.037 \mathrm{E}-10$ & 8.554E-09 \\
\hline & Renal cell carcinoma & 13 & 66 & 9.5059 & 3.543E-09 & $1.240 \mathrm{E}-07$ \\
\hline & PI3K-Akt signaling pathway & 28 & 347 & 3.8942 & $5.365 \mathrm{E}-09$ & $1.610 \mathrm{E}-07$ \\
\hline & Pathways in cancer & 27 & 327 & 3.9848 & 6.399E-09 & $1.680 \mathrm{E}-07$ \\
\hline & Colorectal cancer & 12 & 62 & 9.3408 & $1.948 \mathrm{E}-08$ & $4.545 \mathrm{E}-07$ \\
\hline & Regulation of actin cytoskeleton & 21 & 215 & 4.7138 & 2.194E-08 & 4.607E-07 \\
\hline & TGF-beta signaling pathway & 13 & 81 & 7.7455 & 4.888E-08 & 8.699E-07 \\
\hline & HTLV-I infection & 23 & 268 & 4.1418 & 4.971E-08 & 8.699E-07 \\
\hline & Circadian entrainment & 14 & 97 & 6.9655 & $5.551 \mathrm{E}-08$ & 8.967E-07 \\
\hline & Melanogenesis & 14 & 101 & 6.6896 & 9.433E-08 & $1.415 \mathrm{E}-06$ \\
\hline & Chronic myeloid leukemia & 12 & 73 & 7.9333 & $1.346 \mathrm{E}-07$ & $1.884 \mathrm{E}-06$ \\
\hline & mTOR signaling pathway & 11 & 60 & 8.8478 & $1.547 \mathrm{E}-07$ & $2.031 \mathrm{E}-06$ \\
\hline & HIF-1 signaling pathway & 14 & 106 & 6.3741 & $1.767 \mathrm{E}-07$ & $2.183 \mathrm{E}-06$ \\
\hline & Wnt signaling pathway & 16 & 143 & 5.3998 & $2.141 \mathrm{E}-07$ & $2.498 \mathrm{E}-06$ \\
\hline & Endocytosis & 19 & 204 & 4.4949 & 2.489E-07 & $2.751 \mathrm{E}-06$ \\
\hline & Viral carcinogenesis & 19 & 207 & 4.4297 & $3.140 \mathrm{E}-07$ & $3.297 \mathrm{E}-06$ \\
\hline & Cholinergic synapse & 14 & 113 & 5.9792 & $4.010 \mathrm{E}-07$ & $4.010 \mathrm{E}-06$ \\
\hline & Amphetamine addiction & 11 & 70 & 7.5838 & 8.093E-07 & $7.725 \mathrm{E}-06$ \\
\hline & Insulin signaling pathway & 15 & 140 & 5.1708 & 9.843E-07 & 8.987E-06 \\
\hline & ErbB signaling pathway & 12 & 88 & 6.5810 & $1.132 \mathrm{E}-06$ & $9.903 \mathrm{E}-06$ \\
\hline & Prostate cancer & 12 & 89 & 6.5071 & $1.284 \mathrm{E}-06$ & $1.078 \mathrm{E}-05$ \\
\hline & $\mathrm{T}$ cell receptor signaling pathway & 13 & 108 & 5.8092 & $1.605 \mathrm{E}-06$ & $1.296 \mathrm{E}-05$ \\
\hline & Chemokine signaling pathway & 17 & 192 & 4.2731 & 2.494E-06 & $1.940 \mathrm{E}-05$ \\
\hline & Pancreatic cancer & 10 & 66 & 7.3122 & 4.108E-06 & $3.081 \mathrm{E}-05$ \\
\hline & Endometrial cancer & 9 & 52 & 8.3528 & 4.485E-06 & $3.248 \mathrm{E}-05$ \\
\hline & Circadian rhythm & 7 & 31 & 10.8976 & $1.092 \mathrm{E}-05$ & $7.645 \mathrm{E}-05$ \\
\hline & GnRH signaling pathway & 11 & 92 & 5.7703 & $1.330 \mathrm{E}-05$ & $9.010 \mathrm{E}-05$ \\
\hline & Dopaminergic synapse & 13 & 131 & 4.7892 & $1.463 \mathrm{E}-05$ & $9.601 \mathrm{E}-05$ \\
\hline & Phosphatidylinositol signaling system & 10 & 81 & 5.9581 & $2.766 \mathrm{E}-05$ & $1.760 \mathrm{E}-04$ \\
\hline & Estrogen signaling pathway & 11 & 100 & 5.3087 & $3.017 \mathrm{E}-05$ & $1.839 \mathrm{E}-04$ \\
\hline & Glioma & 9 & 65 & 6.6823 & $3.065 \mathrm{E}-05$ & $1.839 \mathrm{E}-04$ \\
\hline & Cocaine addiction & 8 & 50 & 7.7217 & 3.277E-05 & $1.911 \mathrm{E}-04$ \\
\hline & Insulin secretion & 10 & 87 & 5.5472 & $5.269 \mathrm{E}-05$ & $2.990 \mathrm{E}-04$ \\
\hline & Apoptosis & 10 & 88 & 5.4842 & $5.835 \mathrm{E}-05$ & $3.225 \mathrm{E}-04$ \\
\hline & Long-term potentiation & 9 & 71 & 6.1176 & 6.393E-05 & $3.442 \mathrm{E}-04$ \\
\hline & Acute myeloid leukemia & 8 & 57 & 6.7734 & 8.877E-05 & 4.635E-04 \\
\hline & Hepatitis C & 12 & 133 & 4.3544 & $9.049 \mathrm{E}-05$ & 4.635E-04 \\
\hline & Hippo signaling pathway & 13 & 156 & 4.0217 & 9.772E-05 & $4.886 \mathrm{E}-04$ \\
\hline & Alcoholism & 14 & 180 & 3.7536 & $1.046 \mathrm{E}-04$ & 4.993E-04 \\
\hline & $\begin{array}{l}\text { Transcriptional misregulation } \\
\text { in cancer }\end{array}$ & 14 & 180 & 3.7536 & $1.046 \mathrm{E}-04$ & 4.993E-04 \\
\hline & Calcium signaling pathway & 14 & 183 & 3.6921 & $1.256 \mathrm{E}-04$ & $5.864 \mathrm{E}-04$ \\
\hline & Tuberculosis & 14 & 184 & 3.6720 & 1.334E-04 & $6.092 \mathrm{E}-04$ \\
\hline
\end{tabular}


Table VIII. Continued.

\begin{tabular}{|c|c|c|c|c|c|c|}
\hline Regulation & Name & $\begin{array}{l}\text { Diffgene } \\
\text { count }\end{array}$ & $\begin{array}{l}\text { Gene } \\
\text { count }\end{array}$ & Enrichment & P-value & FDR \\
\hline & $\begin{array}{l}\text { Retrograde endocannabinoid } \\
\text { signaling }\end{array}$ & 10 & 103 & 4.6855 & 2.309E-04 & $1.032 \mathrm{E}-03$ \\
\hline & $\begin{array}{l}\text { Chagas disease (American } \\
\text { trypanosomiasis) }\end{array}$ & 10 & 105 & 4.5963 & $2.720 \mathrm{E}-04$ & $1.190 \mathrm{E}-03$ \\
\hline & GABAergic synapse & 9 & 90 & 4.8261 & 4.275E-04 & $1.832 \mathrm{E}-03$ \\
\hline & Non-small cell lung cancer & 7 & 54 & 6.2560 & 4.865E-04 & $2.041 \mathrm{E}-03$ \\
\hline & Osteoclast differentiation & 11 & 135 & 3.9324 & 4.958E-04 & $2.041 \mathrm{E}-03$ \\
\hline & Adherens junction & 8 & 73 & 5.2888 & 5.398E-04 & 2.180E-03 \\
\hline & Morphine addiction & 9 & 93 & 4.6704 & $5.510 \mathrm{E}-04$ & $2.183 \mathrm{E}-03$ \\
\hline & Fc gamma R-mediated phagocytosis & 9 & 94 & 4.6207 & 5.983E-04 & 2.301E-03 \\
\hline & Ubiquitin mediated proteolysis & 11 & 138 & 3.8469 & $6.026 \mathrm{E}-04$ & 2.301E-03 \\
\hline & Gastric acid secretion & 8 & 75 & 5.1478 & $6.534 \mathrm{E}-04$ & $2.450 \mathrm{E}-03$ \\
\hline & B cell receptor signaling pathway & 8 & 76 & 5.0801 & 7.171E-04 & $2.616 \mathrm{E}-03$ \\
\hline & Glutamatergic synapse & 10 & 118 & 4.0899 & $7.226 \mathrm{E}-04$ & $2.616 \mathrm{E}-03$ \\
\hline & $\begin{array}{l}\text { Protein processing in endoplasmic } \\
\text { reticulum }\end{array}$ & 12 & 167 & 3.4678 & 8.172E-04 & 2.909E-03 \\
\hline & Shigellosis & 7 & 61 & 5.5381 & $1.057 \mathrm{E}-03$ & 3.701E-03 \\
\hline & Cell cycle & 10 & 124 & 3.8920 & $1.083 \mathrm{E}-03$ & $3.728 \mathrm{E}-03$ \\
\hline & Thyroid cancer & 5 & 29 & 8.3208 & 1.187E-03 & 4.021E-03 \\
\hline & $\begin{array}{l}\text { Hypertrophic cardiomyopathy } \\
(\mathrm{HCM})\end{array}$ & 8 & 85 & 4.5422 & $1.555 \mathrm{E}-03$ & 5.183E-03 \\
\hline & $\begin{array}{l}\text { Progesterone-mediated oocyte } \\
\text { maturation }\end{array}$ & 8 & 86 & 4.4894 & 1.684E-03 & $5.525 \mathrm{E}-03$ \\
\hline & Jak-STAT signaling pathway & 11 & 158 & 3.3599 & $1.942 \mathrm{E}-03$ & 6.274E-03 \\
\hline & Measles & 10 & 134 & 3.6015 & $2.013 \mathrm{E}-03$ & $6.388 \mathrm{E}-03$ \\
\hline & $\begin{array}{l}\text { Endocrine and other factor- } \\
\text { regulated calcium reabsorption }\end{array}$ & 6 & 49 & 5.9095 & $2.038 \mathrm{E}-03$ & $6.388 \mathrm{E}-03$ \\
\hline & Oocyte meiosis & 9 & 112 & 3.8781 & 2.224E-03 & 6.867E-03 \\
\hline & Salivary secretion & 8 & 90 & 4.2898 & 2.288E-03 & 6.964E-03 \\
\hline & Dilated cardiomyopathy & 8 & 91 & 4.2427 & $2.464 \mathrm{E}-03$ & 7.392E-03 \\
\hline & $\begin{array}{l}\text { RIG-I-like receptor signaling } \\
\text { pathway }\end{array}$ & 7 & 71 & 4.7581 & $2.697 \mathrm{E}-03$ & 7.976E-03 \\
\hline & Legionellosis & 6 & 55 & 5.2648 & 3.804E-03 & $1.110 \mathrm{E}-02$ \\
\hline & $\begin{array}{l}\text { Aldosterone-regulated sodium } \\
\text { reabsorption }\end{array}$ & 5 & 39 & 6.1873 & 4.853E-03 & $1.396 \mathrm{E}-02$ \\
\hline & Influenza A & 11 & 179 & 2.9657 & $5.421 \mathrm{E}-03$ & $1.538 \mathrm{E}-02$ \\
\hline & Dorso-ventral axis formation & 4 & 24 & 8.0435 & 5.593E-03 & $1.566 \mathrm{E}-02$ \\
\hline & $\begin{array}{l}\text { Cytokine-cytokine receptor } \\
\text { interaction }\end{array}$ & 14 & 267 & 2.5305 & $6.010 \mathrm{E}-03$ & $1.661 \mathrm{E}-02$ \\
\hline & Huntington's disease & 11 & 183 & 2.9009 & $6.465 \mathrm{E}-03$ & $1.763 \mathrm{E}-02$ \\
\hline & Inositol phosphate metabolism & 6 & 61 & 4.7470 & $6.563 \mathrm{E}-03$ & $1.767 \mathrm{E}-02$ \\
\hline & Toll-like receptor signaling pathway & 8 & 108 & 3.5749 & 7.514E-03 & $1.997 \mathrm{E}-02$ \\
\hline & Herpes simplex infection & 11 & 188 & 2.8238 & 7.997E-03 & 2.099E-02 \\
\hline & $\begin{array}{l}\text { Vasopressin-regulated water } \\
\text { reabsorption }\end{array}$ & 5 & 45 & 5.3623 & $9.299 \mathrm{E}-03$ & $2.411 \mathrm{E}-02$ \\
\hline & Gap junction & 7 & 89 & 3.7958 & 1.015E-02 & $2.600 \mathrm{E}-02$ \\
\hline & Serotonergic synapse & 8 & 114 & 3.3867 & $1.055 \mathrm{E}-02$ & $2.656 \mathrm{E}-02$ \\
\hline & VEGF signaling pathway & 6 & 67 & 4.3219 & 1.063E-02 & $2.656 \mathrm{E}-02$ \\
\hline & NF-kappa B signaling pathway & 7 & 92 & 3.6720 & 1.224E-02 & $3.024 \mathrm{E}-02$ \\
\hline & Notch signaling pathway & 5 & 48 & 5.0272 & 1.239E-02 & $3.025 \mathrm{E}-02$ \\
\hline & Fc epsilon RI signaling pathway & 6 & 70 & 4.1366 & 1.325E-02 & 3.199E-02 \\
\hline & Lysine degradation & 5 & 49 & 4.9246 & $1.356 \mathrm{E}-02$ & $3.236 \mathrm{E}-02$ \\
\hline
\end{tabular}


Table VIII. Continued.

\begin{tabular}{llrrrr}
\hline \multicolumn{1}{c}{ Name } & $\begin{array}{c}\text { Diffgene } \\
\text { count }\end{array}$ & $\begin{array}{c}\text { Gene } \\
\text { count }\end{array}$ & Enrichment & P-value & FDR \\
\hline Adipocytokine signaling pathway & 6 & 71 & 4.0784 & $1.423 \mathrm{E}-02$ & $3.320 \mathrm{E}-02$ \\
Melanoma & 6 & 71 & 4.0784 & $1.423 \mathrm{E}-02$ & $3.320 \mathrm{E}-02$ \\
Epstein-Barr virus infection & 11 & 204 & 2.6023 & $1.498 \mathrm{E}-02$ & $3.457 \mathrm{E}-02$ \\
RNA degradation & 6 & 72 & 4.0217 & $1.525 \mathrm{E}-02$ & $3.482 \mathrm{E}-02$ \\
Focal adhesion & 11 & 206 & 2.5770 & $1.612 \mathrm{E}-02$ & $3.640 \mathrm{E}-02$ \\
Pertussis & 6 & 75 & 3.8609 & $1.866 \mathrm{E}-02$ & $4.125 \mathrm{E}-02$ \\
Arrhythmogenic right ventricular & 6 & 75 & 3.8609 & $1.866 \mathrm{E}-02$ & $4.125 \mathrm{E}-02$ \\
cardiomyopathy (ARVC) & & & & & \\
Regulation of autophagy & 4 & 34 & 5.6777 & $2.073 \mathrm{E}-02$ & $4.534 \mathrm{E}-02$ \\
SNARE interactions in vesicular & 4 & 36 & 5.3623 & $2.549 \mathrm{E}-02$ & $5.517 \mathrm{E}-02$ \\
transport & & & & & \\
Bladder cancer & 4 & 38 & 5.0801 & $3.091 \mathrm{E}-02$ & $6.625 \mathrm{E}-02$ \\
Natural killer cell mediated & 8 & 140 & 2.7578 & $3.597 \mathrm{E}-02$ & $7.568 \mathrm{E}-02$ \\
$\quad$ cytotoxicity & & & & & \\
Small cell lung cancer & 6 & 86 & 3.3670 & $3.604 \mathrm{E}-02$ & $7.568 \mathrm{E}-02$ \\
Salmonella infection & 6 & 88 & 3.2905 & $4.013 \mathrm{E}-02$ & $8.345 \mathrm{E}-02$ \\
\hline
\end{tabular}

A Pathways probably up-regulated enrichment in miRNA $(-\operatorname{LgP})$

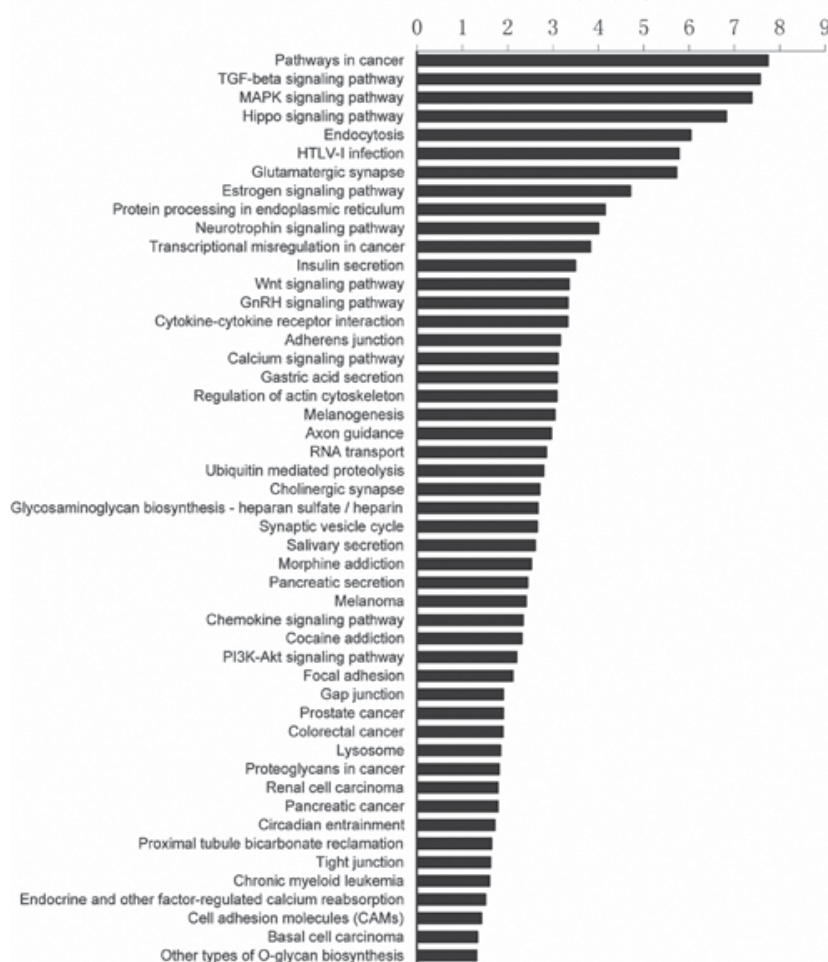

B Pathways probably down-regulated enrichment in miRNA

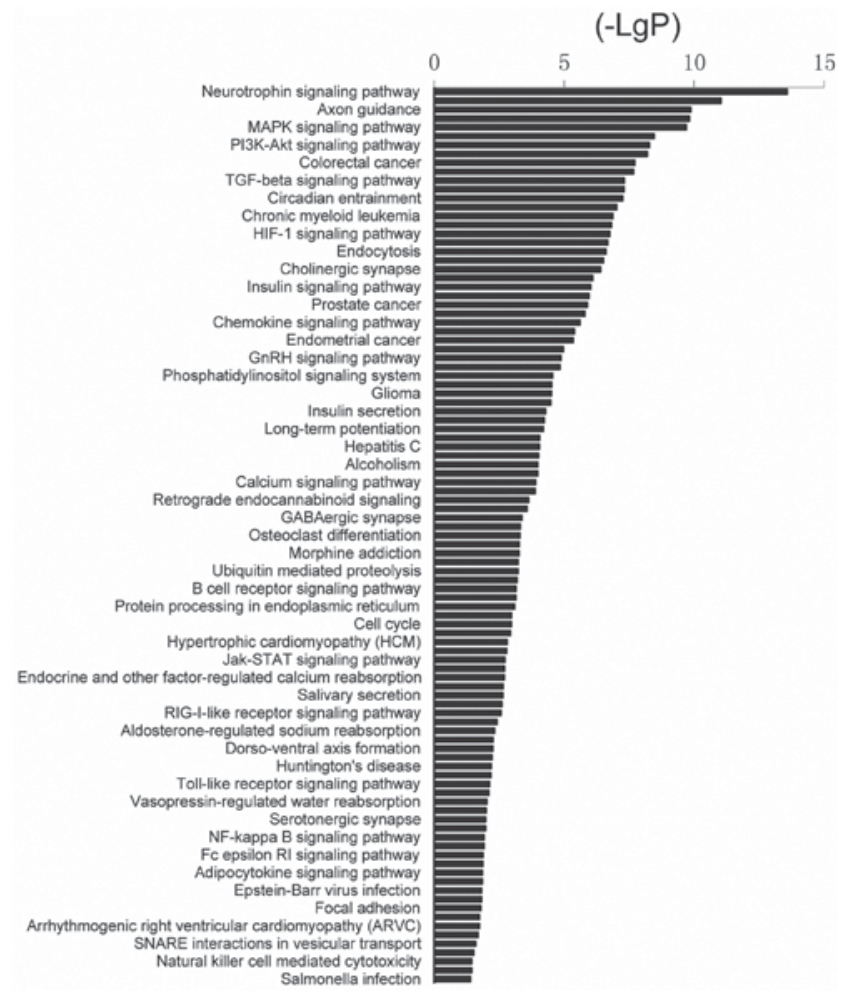

Figure 3. Pathway analysis based on miRNA-targeted genes. (A and B) The histogram of significant pathways targeted by up-regulated (A) and down-regulated miRNA (B) were shown. The vertical axis is the pathway category, and the horizontal axis represents-lg (P-value) of the pathways.

and Fig. 3). Signaling pathways associated with organ size, cell differentiation, cell proliferation and migration, such as transforming growth factor (TGF)- $\beta$, mitogen-activated protein kinase (MAPK), Hippo, PI3K-Akt, Wnt, mTOR, Jak/STAT, NF- $\kappa$ B and Notch, were identified. These data suggested the involvement of these 14 DEMs on the pathology of sIUGR.

miRNA-pathway network analysis. Based on the significantly regulated pathways, we further established miRNA-pathway 

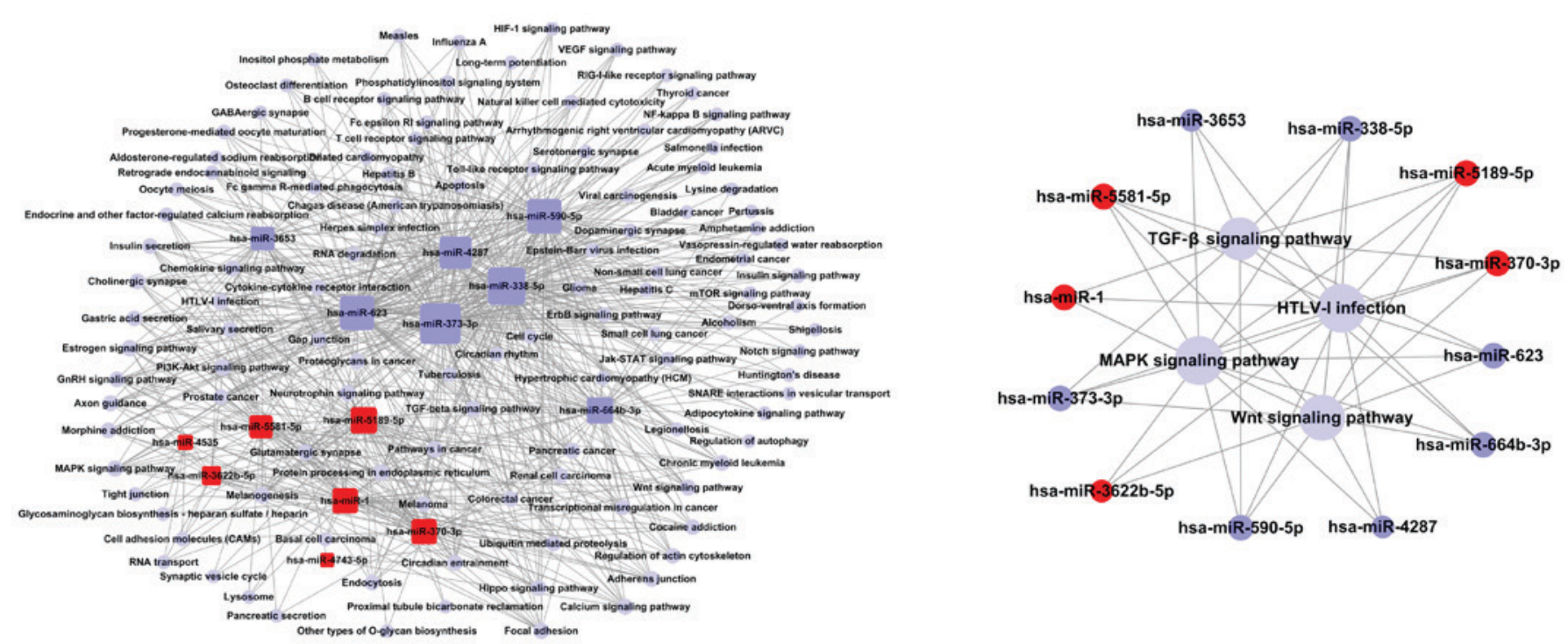

Figure 4. miRNA-pathway network. Red box nodes and blue box nodes represent up-regulated miRNA and down-regulated miRNA, respectively. Blue cycle nodes represent Pathway. Edges show the inhibitory effect of miRNA on Pathway. Left network included all the pathways. When the area of box or circle is larger, the degree of the miRNA or pathway is bigger. Right network extracted from the left network including the key miRNAs and pathways.
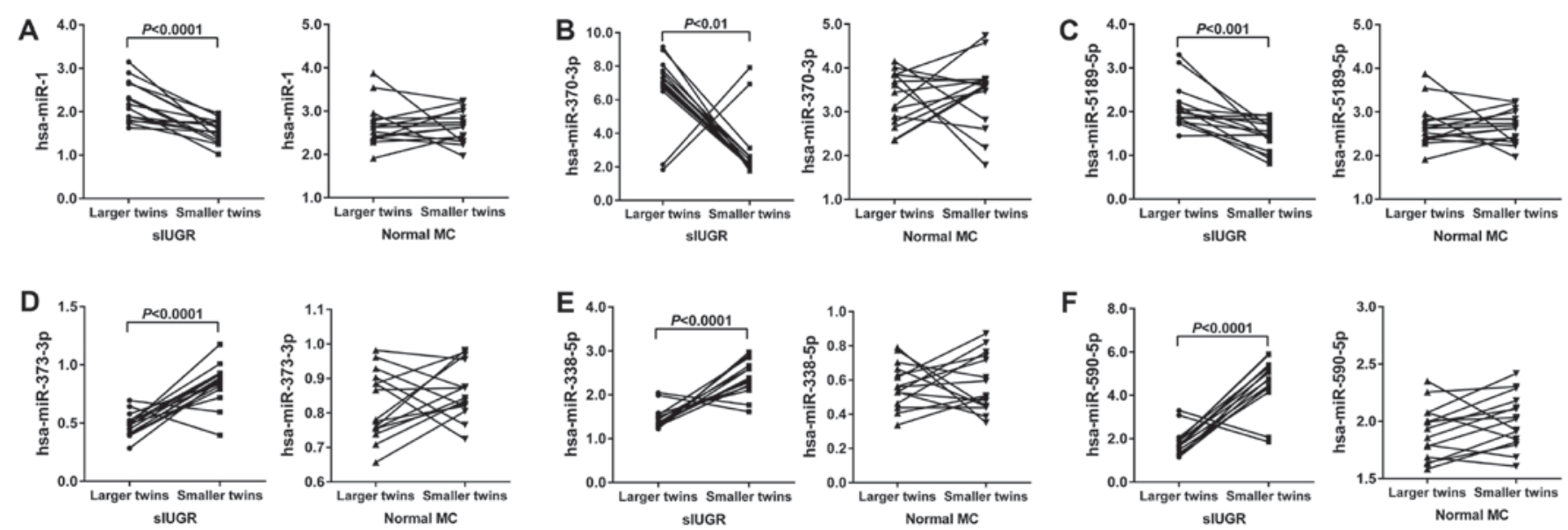

Figure 5. Expression of (A) has-miR-1, (B) has-miR-370-3p, (C) has-miR-5189-5p, (D) has-miR-373-3p, (E) has-miR-338-5p and (F) has-miR-590-5p in placenta tissues from sIUGR and normal NC by qRT-PCR analysis. $\mathrm{n}=15$.

networks to screen the key regulatory functions and the key DEMs (Fig. 4). The top rated five miRNAs included hsa-miR-373-3p, hsa-miR-338-5p, hsa-miR-590-5p, hsa-miR-623 and hsa-miR-4287 (Table IX), all of which were down-regulated in placenta tissues supporting larger twins of sIUGR. The DEMs mainly play vital roles in various biological processes, including HTLV-I infection and signal transduction (TGF- $\beta$, MAPK and Wnt signaling pathways). These networks provided a large amount of information about the regulation of miRNAs in placenta tissues during the development of sIUGR.

Verification of miRNAs microarray with qRT-PCR. We chose three down-regulated miRNAs (has-miR-373-3p, has-miR-338-5p and has-miR-590-5p) and three up-regulated miRNAs (has-miR-1, has-miR-370-3p and has-miR-5189-5p) for the validation analysis. Our validation cohort included 15 cases with sIUGR [larger twin (L3-L17), smaller twin
(S3-S17)] and 15 cases with normal MC [larger twin (N2-N16) and smaller twin (n2-n16)]. The qRT-PCR results showed that the expression changes of these six miRNAs were in the same direction as determined by the miRNA microarray (Fig. 5).

\section{Discussion}

sIUGR MC twin gestations complicated by sIUGR are at high risk of perinatal complications. Recently, some studies have reported that miRNAs are associated with pregnancy-specific diseases (6). Although the pathophysiological insight of sIUGR has been substantially improved, there are few studies on miRNA profiles in the placentas complicated with sIUGR. In this microarray study, we evaluated differential placental miRNA expression in the territory of sIUGR larger twin than in that of corresponding smaller twin. We found 14 placenta miRNAs (7 up-regulated and 
Table IX. The degrees of miRNA-Pathway-networks.

\begin{tabular}{clrl}
\hline Rank & \multicolumn{1}{c}{ miRNAs } & Degree & Feature \\
\hline 1 & hsa-miR-373-3p & 100 & Down \\
2 & hsa-miR-338-5p & 88 & Down \\
3 & hsa-miR-590-5p & 78 & Down \\
4 & hsa-miR-623 & 77 & Down \\
5 & hsa-miR-4287 & 69 & Down \\
6 & hsa-miR-5189-5p & 48 & Up \\
7 & hsa-miR-664b-3p & 48 & Down \\
8 & hsa-miR-1 & 44 & Up \\
9 & hsa-miR-370-3p & 44 & Up \\
10 & hsa-miR-3653 & 39 & Down \\
11 & hsa-miR-5581-5p & 36 & Up \\
12 & hsa-miR-3622b-5p & 22 & Up \\
13 & hsa-miR-4535 & 7 & Up \\
14 & hsa-miR-4743-5p & 4 & Up \\
\hline
\end{tabular}

The degree of each miRNA was the number of pathways regulated by that miRNA.

7 down-regulated) specifically significantly differentially expressed among larger twins of sIUGR cases compared with smaller twins of sIUGR cases. Differentially expressed miRNAs included those that were previously associated with pregnancy-specific diseases, such as preterm delivery and preeclampsia (miR-338, miR-590-5p and miR-1) (24-26), and others that are novel in pregnancy-specific diseases (miR-373-3p, miR-623, miR-4287, miR-664b-3p, miR-3653, miR-5189-5p, miR-370-3p, miR-5581-5p, miR-3622b-5p, miR-4535 and miR-4743-5p). Several of these DEMs have been implicated in tumorigenesis of various types of tumors, such as miR-373-3p in breast, liver, gastric, esophageal, colon, prostate, pancreatic and lung cancer (27), miR-338-5p in colorectal (28) and liver cancer (29), miR-590-5p in cervical cancer (30), miR-623 in lung adenocarcinoma and miR-370-3p in glioma (31). Some of these DEMs have been identified in association with other human diseases. For example, miR-1 has been reported as a biomarker for predicting acute myocardial infarction (32). miR-4743 may serve as biomarker for the diagnosis of Major Depressive Disorder (MDD) (33).

Further, target genes of these DEMs were predicted and the pathway analysis was performed. The target genes are participated in diverse pathophysiological processes including cell organ size, cell differentiation, cell proliferation and cell migration, which may implicated in the pathogenesis of sIGUR. DEMs, including miR-373 (27), miR-338-5p (34), miR-590-5p (30,35,36), miR-623 (37) and miR-370-3p (31), have been reported involved in regulating the proliferation, migration and invasion of cancer cells, which was consistent with our findings. Further studies on the expression pattern and function of these target genes may advance our understanding of the implications of theses DEMs in sIGUR pathogenesis. To reveal miRNA regulation of pathways, miRNA-pathway network was built. Of note, key miRNAs and pathways (TGF- $\beta$, MAPK and Wnt) were identified (Fig. 4B). The TGF- $\beta$ signaling pathway participates in diverse biological processes, including the formation of tissues and organs (38). miR-373 (39) and miR-590-5p (35) exerted their metastasis-inhibiting function via TGF- $\beta$ signaling pathway. Wnt and MAPK signaling pathways are involved in the development of placenta (40). It has been shown that miR-370-3p (31) and miR-590-5p (36) suppressed the growth of glioma and liver cancer cells, respectively, by targeting Wnt/ $\beta$-catenin. miR-623 suppressed the invasion of lung adenocarcinoma cells through inactivating MAPK ERK/JNK (37). These results lay a foundation and provide ideas for future in-depth studies, particularly related to the 14 miRNAs specifically changed in sIUGR.

In summary, we have shown the differential placental miRNA expression associated with sIUGR. In addition, the results of the pathway analysis and miRNA-pathway network analysis represented comprehensive information on the molecular mechanisms of sIUGR from the point of miRNAs. Further experimental studies to evaluate biologic effects of identified miRNAs are warranted.

\section{Acknowledgements}

This study was supported by Scientific Research Project of the Health and family planning commission of Zhejiang Province, China (2014KYA253).

\section{References}

1. Gomes CP, Cho JH, Hood L, Franco OL, Pereira RW and Wang K: A review of computational tools in microRNA discovery. Front Genet 4: 81, 2013.

2. Du T and Zamore PD: Beginning to understand microRNA function. Cell Res 17: 661-663, 2007.

3. Lu M, Zhang Q, Deng M, Miao J, Guo Y, Gao W and Cui Q: An analysis of human microRNA and disease associations. PLoS One 3: e3420, 2008.

4. Miska EA: How microRNAs control cell division, differentiation and death. Curr Opin Genet Dev 15: 563-568, 2005.

5. Bentwich I, Avniel A, Karov Y, Aharonov R, Gilad S, Barad O, Barzilai A, Einat P, Einav U, Meiri E, et al: Identification of hundreds of conserved and nonconserved human microRNAs. Nat Genet 37: 766-770, 2005.

6. Zhao Z, Moley KH and Gronowski AM: Diagnostic potential for miRNAs as biomarkers for pregnancy-specific diseases. Clin Biochem 46: 953-960, 2013.

7. Chang YL, Chang SD, Chao AS, Hsieh PC, Wang CN and Wang TH: Clinical outcome and placental territory ratio of monochorionic twin pregnancies and selective intrauterine growth restriction with different types of umbilical artery Doppler. Prenat Diagn 29: 253-256, 2009.

8. Gratacós E, Lewi L, Muñoz B, Acosta-Rojas R, Hernandez-Andrade E, Martinez JM, Carreras E and Deprest J: A classification system for selective intrauterine growth restriction in monochorionic pregnancies according to umbilical artery Doppler flow in the smaller twin. Ultrasound Obstet Gynecol 30: 28-34, 2007.

9. Weisz B, Hogen L, Yinon Y, Gindes L, Shrim A, Simchen M, Schiff E and Lipitz S: Perinatal outcome of monochorionic twins with selective IUGR compared with uncomplicated monochorionic twins. Twin Res Hum Genet 14: 457-462, 2011.

10. Valsky DV, Eixarch E, Martinez JM, Crispi F and Gratacós E: Selective intrauterine growth restriction in monochorionic twins: Pathophysiology, diagnostic approach and management dilemmas. Semin Fetal Neonatal Med 15: 342-348, 2010. 
11. Lewi L, Cannie M, Blickstein I, Jani J, Huber A, Hecher K, Dymarkowski S, Gratacós E, Lewi P and Deprest J: Placental sharing, birthweight discordance, and vascular anastomoses in monochorionic diamniotic twin placentas. Am J Obstet Gynecol 197: 587.e1-8, 2007.

12. Lewi L, Gucciardo L, Huber A, Jani J, Van Mieghem T, Doné E, Cannie M, Gratacós E, Diemert A, Hecher K, et al: Clinical outcome and placental characteristics of monochorionic diamniotic twin pairs with early- and late-onset discordant growth Am J Obstet Gynecol 199: 511.e1-7, 2008.

13. Nevo O, Many A, Xu J, Kingdom J, Piccoli E, Zamudio S, Post M, Bocking A, Todros T and Caniggia I: Placental expression of soluble fms-like tyrosine kinase 1 is increased in singletons and twin pregnancies with intrauterine growth restriction. J Clin Endocrinol Metab 93: 285-292, 2008.

14. Yinon Y, Ben Meir E, Berezowsky A, Weisz B, Schiff E, Mazaki-Tovi S and Lipitz S: Circulating angiogenic factors in monochorionic twin pregnancies complicated by twin-to-twin transfusion syndrome and selective intrauterine growth restriction. Am J Obstet Gynecol 210: 141.e1-7, 2014.

15. Chu S, Mao Q, Shapiro S and De Paepe ME: Placental endoglin levels in diamniotic-monochorionic twin gestations: Correlation with clinical and placental characteristics. Placenta 34: 261-268, 2013.

16. Shi X, He Z, Gao Y, Luo Y, Gou C and Fang Q: Placental expression of PHLDA2 in selective intrauterine growth restriction in monozygotic twins. Placenta 35: 428-430, 2014.

17. Loke YJ, Galati JC, Morley R, Joo EJ, Novakovic B, Li X, Weinrich B, Carson N, Ollikainen M, Ng HK, et al: Association of maternal and nutrient supply line factors with DNA methylation at the imprinted IGF2/H19 locus in multiple tissues of newborn twins. Epigenetics 8: 1069-1079, 2013.

18. Enright AJ, John B, Gaul U, Tuschl T, Sander C and Marks DS MicroRNA targets in Drosophila. Genome Biol 5: R1, 2003.

19. Lewis BP, Shih IH, Jones-Rhoades MW, Bartel DP and Burge CB Prediction of mammalian microRNA targets. Cell 115: 787-798, 2003.

20. Kanehisa M, Goto S, Kawashima S, Okuno Y and Hattori M: The KEGG resource for deciphering the genome. Nucleic Acids Res 32 (Database Issue): D277-D280, 2004

21. Yi M, Horton JD, Cohen JC, Hobbs HH and Stephens RM: WholePathwayScope: A comprehensive pathway-based analysis tool for high-throughput data. BMC Bioinformatics 7: 30, 2006.

22. Draghici S, Khatri P, Tarca AL, Amin K, Done A, Voichita C, Georgescu C and Romero R: A systems biology approach for pathway level analysis. Genome Res 17: 1537-1545, 2007.

23. Bao Y, Gao Y, Jin Y, Cong W, Pan X and Cui X: MicroRNA expression profiles and networks in mouse lung infected with H1N1 influenza virus. Mol Genet Genomics 290: 1885-1897, 2015.

24. Montenegro D, Romero R, Kim SS, Tarca AL, Draghici S, Kusanovic JP, Kim JS, Lee DC, Erez O, Gotsch F, et al: Expression patterns of microRNAs in the chorioamniotic membranes: A role for microRNAs in human pregnancy and parturition. J Pathol 217: 113-121, 2009.

25. Enquobahrie DA, Abetew DF, Sorensen TK, Willoughby D, Chidambaram K and Williams MA: Placental microRNA expression in pregnancies complicated by preeclampsia. Am J Obstet Gynecol 204: 178.e12-21, 2011.
26. Yang Q, Lu J, Wang S, Li H, Ge Q and Lu Z: Application of next-generation sequencing technology to profile the circulating microRNAs in the serum of preeclampsia versus normal pregnant women. Clin Chim Acta 412: 2167-2173, 2011.

27. Wei F, Cao C, Xu X and Wang J: Diverse functions of miR-373 in cancer. J Transl Med 13: 162, 2015

28. Yong FL, Law CW and Wang CW: Potentiality of a triple microRNA classifier: miR-193a-3p, miR-23a and miR-338-5p for early detection of colorectal cancer. BMC Cancer 13: 280, 2013.

29. Chen Y, Chen J, Liu Y, Li S and Huang P: Plasma miR-15b-5p, miR-338-5p, and miR-764 as biomarkers for hepatocellular carcinoma. Med Sci Monit 21: 1864-1871, 2015.

30. Chu Y, Ouyang Y, Wang F, Zheng A, Bai L, Han L, Chen Y and Wang H: MicroRNA-590 promotes cervical cancer cell growth and invasion by targeting CHL1. J Cell Biochem 115: 847-853, 2014.

31. Peng Z, Wu T, Li Y, Xu Z, Zhang S, Liu B, Chen Q and Tian D: MicroRNA-370-3p inhibits human glioma cell proliferation and induces cell cycle arrest by directly targeting $\beta$-catenin. Brain Res 1644: 53-61, 2016.

32. Ai J, Zhang R, Li Y, Pu J, Lu Y, Jiao J, Li K, Yu B, Li Z, Wang R, et al: Circulating microRNA-1 as a potential novel biomarker for acute myocardial infarction. Biochem Biophys Res Commun 391: 73-77, 2010.

33. Qiao-li Z, Jim L, Xin-yang S, Wei Guo, Lin Zhao, Hui-min Fan, Ai-fang Zhong, Wei Niu, Yun-hua Dai, Li-yi Zhang, et al: A preliminary analysis of association between plasma microRNA expression alteration and symptomatology improvement in Major Depressive Disorder (MDD) patients before and after antidepressant treatment. Eur J Psychiatry 28: 252-264, 2014.

34. Ju JA, Huang CT, Lan SH, Wang TH, Lin PC, Lee JC, Tian YF and Liu HS: Characterization of a colorectal cancer migration and autophagy-related microRNA miR-338-5p and its target gene PIK3C3. Biomark Genomic Med 5: 74-78, 2013.

35. Jiang X, Xiang G, Wang Y, Zhang L, Yang X, Cao L, Peng H, Xue P and Chen D: MicroRNA-590-5p regulates proliferation and invasion in human hepatocellular carcinoma cells by targeting TGF- $\beta$ RII. Mol Cells 33: 545-551, 2012.

36. Shan X, Miao Y, Fan R, Qian H, Chen P, Liu H, Yan X, Li J and Zhou F: MiR-590-5P inhibits growth of HepG2 cells via decrease of S100A10 expression and inhibition of the Wnt pathway. Int J Mol Sci 14: 8556-8569, 2013.

37. Wei S, Zhang ZY, Fu SL Xie JG, Liu XS, Xu YJ, Zhao JP and Xiong WN: Hsa-miR-623 suppresses tumor progression in human lung adenocarcinoma. Cell Death Dis 8: e2829, 2017.

38. Massagué J and Chen YG: Controlling TGF-beta signaling. Genes Dev 14: 627-644, 2000.

39. Keklikoglou I, Koerner C, Schmidt C, Zhang JD, Heckmann D, Shavinskaya A, Allgayer H, Gückel B, Fehm T, Schneeweiss A, et al: MicroRNA-520/373 family functions as a tumor suppressor in estrogen receptor negative breast cancer by targeting NF- $\kappa$ B and TGF- $\beta$ signaling pathways. Oncogene 31 : 4150-4163, 2012.

40. Rossant J and Cross JC: Placental development: Lessons from mouse mutants. Nat Rev Genet 2: 538-548, 2001 